\title{
AN EIGENVALUE PROBLEM FOR NONLINEAR ELLIPTIC PARTIAL DIFFERENTIAL EQUATIONS
}

\author{
BY \\ MELVYN S. BERGER
}

Introduction. In this work a concrete nonlinear problem in the theory of elliptic partial differential equations is studied by the methods of functional analysis on Sobolev spaces. More specifically, let $G$ be a bounded domain in Euclidean $N$-space $R^{N}$, and let $A$ be a formally self-adjoint linear elliptic differential operator of order $2 m$ defined on $G$. Then we consider the boundary value problem

$$
\begin{aligned}
A u-\lambda f(u, x) & =0, \\
\left.u\right|_{\partial G} & =\left.D u\right|_{\partial G}=\cdots=\left.D^{m-1} u\right|_{\partial G}=0,
\end{aligned}
$$

where $\lambda$ is a real number and $f(t, x)$ is a real-valued function defined on $R^{1} \times G$ with $f(0, x) \equiv 0$.

If $f(u, x)=u$, the study of the boundary value problem (1) forms the foundation of the spectral analysis of $A$, a problem of great importance both in mathematics and its applications. If $f(u, x)$ does not depend on $u$ in a linear manner, one enters the relatively uncharted world of nonlinear functional analysis.

We shall be concerned with the existence of real-valued nontrivial solutions of (1), i.e. eigenfunctions. There are basically two different approaches to such nonlinear existence problems: first the methods of fixed point theory and other topological principles used with success in the study of elliptic partial differential equations since the pioneering work of S. Bernstein and J. Schauder; second the variational method, dating back to Gauss, Dirichlet and Riemann, and currently, in combination with the new methods of Sobolev spaces, undergoing a rapid development. Throughout this study we shall rely on this latter approach.

For second order operators $A$, one of the first treatments of boundary value problems of the type considered here was given by A. Hammerstein [17], in 1930, as an application of his study of nonlinear integral equations.

The approach used in this dissertation is based on a direct study of elliptic differential operators without recourse to integral equations and Green's functions. By focusing attention on the so-called generalized solutions of (1), we are able to use a variety of Hilbert spaces in our study and to eliminate the auxiliary analytic machinery of a priori estimates, and smoothness properties on the domain $G$.

The following questions will occupy our attention in this study.

(i) (Existence Problem). Under what restrictions on the function $f(t, x)$ does

Received by the editors August 6, 1964. 
the boundary value problem (1) possess a real-valued generalized eigenfunction with associated real eigenvalue $\lambda$ ?

(ii) Is there a generalized eigenfunction for (1) whose associated eigenvalue $\lambda$ is the first eigenvalue for (1) in some sense?

(iii) (Regularity Problem). Under what condition on (1) is every generalized eigenfunction sufficiently smooth to be an eigenfunction for (1) in the classical sense?

(iv) What features of the boundary value problem (1) distinguish it from its linear analogue when $f(u, x)=g(x) u$ ?

(v) In the case of second order operators $A$, what are the positivity properties of the generalized and classical eigenfunctions obtained? Can the above results be sharpened for ordinary differential equations? For bounded domains $G$ in $R^{2}$ ?

The basic notations and results used throughout are summarized in $\S$ I. In $\S I I$ we state the existence problem in full detail and show how its solution can be reduced to consideration of a certain operator equation in the Hilbert space $\mathscr{W}_{2}^{(m)}(G)$.

Tentative solutions of this operator equation are normalized, but not, as in the linear case, by the requirement that they be of norm 1 . Rather, they lie on an "energy level" set $\partial M_{R}$ in $\mathscr{W}_{2}^{(m)}(G)$. The study and definition of these sets are taken up in §III. From the point of view of the geometry of Banach spaces, the energy level $\partial M_{R}$ is the boundary of a closed convex but unbounded set in $\mathscr{W}_{2}^{(m)}(G)$. Surprisingly however, $\partial M_{R}$ is both weakly closed and bounded away from 0 .

In $\S I V$, the variational problem $\inf a(u, u)$ over the energy level $\partial M_{R}$ is considered, where $a(u, v)$ is the associated Dirichlet form for the elliptic operator $A$. This variational problem is solved by the direct method of the calculus of variations, and its solution is also a generalized eigenfunction for the boundary value problem (1). This method is a complete analogue of the linear case (cf. Riesz and Nagy [31]). The most important restriction on the function $f(t, x)$ is a polynomial growth condition similar to that arising in the studies of Višik [37] and Brow$\operatorname{der}[7]$.

By imposing natural smoothness restrictions on the boundary value problem (1) and retaining the growth condition on $f(t, x)$ mentioned above, we show that any generalized eigenfunction of (1) is actually an eigenfunction in the classical sense. This is the main result of $\S \mathrm{V}$. Its proof is based on a powerful bootstrap procedure developed by $\mathrm{F}$. E. Browder using the $L_{P}$ regularity theory for nonhomogeneous linear elliptic differential equations.

The investigation of one distinctively nonlinear feature of (1) is the subject of $\S \mathrm{VI}$. In particular we study the dependence of the eigenvalue $\lambda$, characterized in $\S I V$, on the normalization constant $R$. In sharp contrast to the linear situation, in many cases the set of numbers $\{\lambda(R) \mid 0<R<\infty\}$ contains subsequences tending both to 0 and $+\infty$. 
In §VII, we sharpen the results on the existence problem. First, for second order operators $A$, we obtain a partial extension of the Courant Nodal Line Theorem to a nonlinear context. In case $2 m>N$, we show the existence theorem of $\S \mathrm{IV}$ holds without growth restrictions on $f(t, x)$ (cf. M. Golomb [15]). Finally for second order operators $A$, in $R^{2}$, we mention N. Levinson's result [23] on exponential rates of growth for the function $f(t, x)$.

Previous studies of nonlinear eigenvalue problems fall into several categories. First, there are special studies for ordinary second order differential equations such as Nehari [27], Ljusternik [25] and Pimbley [30]. Secondly, there are general studies in abstract function spaces, of which the works Banach [3], KreinRutman [21], Golomb [15], Ljusternik [26] and Schaeffer [32] are representative. Some of the results obtained apply to partial differential equations but only at the cost of placing extremely strong restrictions on the function $f(t, x)$. For example in Golomb [15], $|f(t, x)|$ must be bounded above by a linear function in $t$. Finally there are studies directly applicable to partial differential equations. Representative works are Levinson [23], Vainberg [35], [36] and Duff [11]. The present work extends Levinson's study from $R^{2}$ to $R{ }^{N}$ and from the Laplace operator to a large class of elliptic operators of order $2 m$. Further, by replacing a study of Green's functions with Garding's inequality and Sobolev's Imbedding Theorem we are able to fit Vainberg's studies into a unified pattern.

In [11], Duff proves the existence of a real positive solution $u_{1}(u)$ for the second order boundary value problem

$$
\begin{aligned}
A u-\lambda F(u, x) & =0, \quad F(u, x) \geqq \delta>0, \\
\left.u\right|_{\partial G} & =g(x) .
\end{aligned}
$$

Our study gives conditions under which (2) has still another distinct real positive solution $u_{2}(x)$ with the same number $\lambda$, for setting $v(x)=u_{2}(x)-u_{1}(x)$, the equation

$$
\begin{aligned}
A v-\lambda\left[F\left(v+u_{1}, x\right)-F\left(u_{1}, x\right)\right] & =0, \\
\left.v\right|_{\partial G} & =0,
\end{aligned}
$$

is a boundary value problem of type (1).

This research represents part of a dissertation submitted to the Graduate School of Yale University in candidacy for the degree of Doctor of Philosophy. The author extends a most hearty thanks to Professor F. E. Browder for constant encouragement and numerous helpful suggestions. This research was partially supported by the National Science Foundation (NSF-GP-2283) and (NSFGP-2280).

I. Preliminaries. In this section we set down the basic terminology and auxiliary results needed in the present study.

I.1. Domains in $R^{N}$. Let $G$ be a fixed bounded domain in Euclidean $N$-space, 
$R^{N}$, with boundary $\partial G$ and closure $\bar{G}=G \cup \partial G$. A general point of $G$ will be denoted by $x=\left(x_{1}, \cdots, x_{N}\right) . G$ is said to be of class $C^{m}$, if the boundary $\partial G$ can be covered by a finite number of open sets, each admitting a representation of the form

$$
x_{k}=g_{k}\left(x_{1}, x_{2}, \cdots, x_{k-1}, x_{k+1}, \cdots, x_{N}\right), \quad k=1, \cdots N,
$$

where the functions $g_{k}$ have continuous derivatives of all orders up to and including order $m$. All functions defined on $G$ will be assumed to be real-valued. Integration over $G$ will always be taken with respect to Lebesgue $N$-dimensional measure.

I.2. Differential operators. We proceed to discuss differential operators defined on $G$. (All derivatives are taken in the generalized sense of L. Schwartz [33].) The following notation is very convenient: the elementary differential operators are written $D_{j}=\partial / i \partial x_{j}(1 \leqq j \leqq N)$ and for any $N$-tuple of nonnegative integers $\alpha=\left(\alpha_{1}, \alpha_{2}, \cdots, \alpha_{N}\right)$, the corresponding differential operator of order $|\alpha|=\alpha_{1}+\alpha_{2} \cdots+\alpha_{N}$ is written $D^{\alpha}=D_{1}^{\alpha_{1}} D_{2}^{\alpha_{2}} \cdots D_{N}^{\alpha_{N}}$.

Thus, in this notation, a general real linear differential operator of order $2 m$ is written: $A u=\sum_{|\alpha| \leqq 2 m} a_{\alpha}(x) D^{\alpha} u$, where $a_{\alpha}(x)$ are real-valued functions defined on $G$. The formal adjoint $A^{1}$ of $A$ is defined as the differential operator $A^{1} u=\sum_{|\alpha| \leqq 2 m} D^{\alpha}\left[a_{\alpha}(x) u\right]$. If the differential operators $A$ and $A^{1}$ are identical $A$ is called formally self-adjoint. An operator $A$ is said to be in divergence form, if it can be written $A u=\sum_{|\alpha|,|\beta| \leqq m} D^{\alpha}\left[a_{\alpha \beta}(x) D^{\beta} u\right]$. In particular, if $a_{\alpha \beta}(x)=a_{\beta \alpha}(x)$, $A$ is formally self-adjoint.

It is useful to classify differential operators by their properties which remain invariant under differentiable coordinate transformations $x=\left(x_{1}, \cdots, x_{N}\right) \rightarrow x^{1}$ $=\left(x_{1}^{1}, \cdots, x_{N}^{1}\right)$. For linear differential operators $A$, the so-called homogeneous characteristic form, having simple transformation properties, is a valuable classifying device.

The homogeneous characteristic form of $A$ is the multinomial $a(x, \xi)$ $=\sum_{|\alpha|=2 m} a_{\alpha}(x) \xi^{\alpha}$, where $\xi=\left(\xi_{1}, \xi_{2}, \cdots, \xi_{N}\right)$ is an $N$-vector of real indeterminates $\xi_{1}, \cdots, \xi_{N}$, and $\xi^{\alpha}=\xi_{1}^{\alpha_{1}} \xi_{2}^{\alpha_{2}} \cdots \xi_{N}^{\alpha_{N}}$. Properties of $a(x, \xi)$ invariant under nonsingular real linear transformations are invariant under all differentiable coordinate transformations. Thus, we say the linear differential operator $A$ is elliptic in $G$ if $a(x, \xi) \neq 0$ for any $x \in G$, and any nonzero $\xi \in R^{N}$. The linear differential operator $A$ is uniformly elliptic in $G$, if there is a constant $c_{0}>0$, such that $a(x, \xi) \geqq c_{0}|\xi|^{2 m}$ for any $x \in G$ and all $\xi \in R^{N}$, where $|\xi|=\left(\xi_{1}^{2}+\cdots+\xi_{N}^{2}\right)^{1 / 2}$ is the length of the real $N$-vector $\xi$.

I.3. Function spaces. Our study of elliptic differential operators will be expressed in the language of functional analysis. In particular, three special classes of Banach spaces enter the theory of elliptic differential equations in a natural manner. (We recall a Banach space is a normed vector space, complete with respect to the norm.)

(1) $C^{m}(\bar{G})$ spaces. (a) $C^{m}(G)$ is the collection of functions $u(x)$, such that $u$ and all its derivatives of order $\leqq m$ are defined and continuous on $G$. 
(b) $C^{m}(\bar{G})$ is the collection of functions in $C^{m}(\bar{G})$ such that $D^{\alpha} u$ for $|\alpha| \leqq m$ are uniformly bounded on $G$. $C^{m}(\bar{G})$ is a Banach space with respect to the norm $\|u\|=\Sigma_{|\alpha| \leqq m} \sup _{G}\left|D^{\alpha} u(x)\right|$.

(c) $C_{0}^{m}(G)$ is the collection of functions in $C^{m}(G)$ which vanish outside a compact subset of $G$.

If $m>l, C^{l}(\bar{G}) \supset C^{m}(\bar{G})$ in the sense that each function $u(x)$ in $C^{m}(\bar{G})$ is also in $C^{l}(\bar{G})$, and convergence with respect to the $C^{m}(\bar{G})$ norm implies convergence with respect to the $C^{l}(\bar{G})$ norm. Further, the Arzela-Ascoli theorem shows any bounded set in $C^{m}(\bar{G})$ is a precompact subset of $C^{l}(\bar{G})$. Thus the imbedding $C^{m}(\bar{G}) \rightarrow C^{l}(\bar{G})$ is a compact linear transformation which is one-to-one.

We formalize the notion of imbedding as follows. Let $X$ and $X_{1}$ be two Banach spaces; then $X$ is imbedded in $X_{1}$ (we write $X \subset X_{1}$ ) if: (1) every element $y \in X$ is also an element of $X_{1}$, and (2) every strongly convergent sequence in $X$ is also a strongly convergent sequence in $X_{1}$. Thus the imbedding operator $i: X \rightarrow X_{1}$ defined by $i(y)=y$ is a continuous linear mapping between two Banach spaces and hence bounded, i.e. there is a positive number $k$ such that $\|y\|_{X_{1}} \leqq k\|y\|_{X}$, for all $y \in X$. The imbedding $X \rightarrow X_{1}$ is compact if the linear operator $i$ is compact.

(2) $C^{m, \gamma}(G)$ spaces (Hölder spaces). (a) A function $u(x)$ defined on $G$ is said to satisfy a Hölder condition with exponent $\gamma(0<\gamma<1)$, if there is a positive constant $k$ such that $|u(x)-u(y)| \leqq k|x-y|^{\gamma}$ for any $x, y \in G$.

(b) $C^{m, \gamma}(G)$ is the collection of functions in $C^{m}(\bar{G})$ whose derivatives of all orders $\leqq m$ satisfy a Hölder condition of exponent $\gamma \cdot C^{m, \gamma}(G)$ is a Banach space with respect to the norm

$$
\|u\|=\|u\|_{C^{m^{\prime}(G)}}+\sum_{|\alpha| \leqq m} \sup _{x, y \in G}\left\{\frac{\left|D^{\alpha} u(x)-D^{\alpha} u(y)\right|}{|x-y|^{\gamma}}\right\} .
$$

We note that $C^{m, \gamma}(G) \subset C^{m}(\bar{G})$, and again, by the Arzela-Ascoli theorem the imbedding $C^{m, \gamma}(G) \rightarrow C^{m}(\bar{G})$ is compact.

The subject of elliptic differential equations based on functional analysis and the $C^{m, \gamma}(G)$ spaces was first extensively studied by the Polish mathematician J. Schauder, in the decade prior to the Second World War.

(3) $W_{p}^{m}(G)$ spaces (Sobolev spaces). First, we define the Lebesgue spaces $L_{p}(G) \equiv W_{p}^{0}(G), 1 \leqq p<\infty$. (a) $L_{p}(G)$ is the collection of equivalence classes a.e. of functions $u(x)$ defined on $G$ such that $\int_{G}|u(x)|^{p}<\infty$, for fixed $p$ with $1 \leqq p<\infty . L_{p}(G)$ is a Banach space with respect to the norm $\|u\|^{p}=\int_{G}|u(x)|^{p}$ $(1 \leqq p<\infty)$.

(b) $L_{2}(G)$ is a Hilbert space (i.e. a vector space with inner product, complete with respect to the norm $\|u\|=\sqrt{ }(u, u)$ with respect to the inner product $(u, v)=\int_{G} u(x) v(x)$. For $1<p<\infty, L_{p}(G)$ is a separable reflexive Banach space, with conjugate space $L_{q}(G)$, where $1 / p+1 / q=1$. Further if $u \in L_{p}(G)$ and $v \in L_{q}(G)$ then by Hölder's Inequality $u v$ is integrable over $G$, and 


$$
\int_{G}|u v| \leqq\|u\|_{L_{p}}\|v\|_{L} .
$$

If $G$ is a bounded domain and $1 \leqq p_{1} \leqq p<\infty, L_{p}(G) \subset L_{p_{1}}(G)$. Hence $\|u\|_{L_{p 1}(G)} \leqq k_{G}\|u\|_{L_{p}(G)}$, for all $u \in L_{p}(G)$.

(c) $W_{p}^{m}(G)$ is the collection of functions on $L_{p}(G)$ such that $D^{\alpha} u$, for all $\mid \alpha \leqq m$, again lies in $L_{p}(G) . W_{p}^{m}(G)$ is a Banach space with respect to the norm

$$
\|u\|_{m, p}^{p}=\sum_{|\alpha| \leqq m}\left\|D^{\alpha} u\right\|_{L_{p}(G)}^{p} .
$$

(d) $W_{2}^{m}(G)$ is a Hilbert space with respect to the inner product $\langle u, v\rangle_{m, 2}=\sum_{\alpha \mid \leqq m}\left(D^{\alpha} u, D^{\alpha} v\right)_{L_{2}(G)}$.

(e) $\mathscr{W}_{2}^{m}(G)$ is the closure of $C_{0}^{\infty}(G)$ in $W_{2}^{(m)}(G) . \mathscr{W}_{2}^{m}(G)$ is closed linear subspace of $W_{2}^{(m)}(G)$, and thus can be regarded itself as a Hilbert space.

I.4. Sobolev's Imbedding Theorem and Corollaries. The following basic result, known as Sobolev's Imbedding Theorem, gives conditions under which $W_{p}^{m}(G)$ can be imbedded in various Banach spaces in a continuous and, sometimes compact manner.

TheOREM I.4.1 (SoboleV-KondRaChov). Suppose $G$ is a bounded domain of class $C^{1}$ in $R^{N}$. Then

(i) $W_{p}^{m}(G) \subset W_{r}^{j}(G)$, for any number $r$, satisfying the relation

$$
\frac{1}{p}-\frac{(m-j)}{N} \leqq \frac{1}{r} \leqq \frac{1}{p i}
$$

Hence there is a number $k_{1}>0$ such that

$$
\|u\|_{j, r} \leqq k_{1}\|u\|_{m, p}, \quad \text { for any } u \in W_{p}^{m}(G) .
$$

The imbedding is compact if $1 / p-(m-j) / N<1 / r \leqq 1 / p$.

(ii) $W_{p}^{m}(G) \subset C^{j}(\bar{G})$, for any number $j$, satisfying the relation

$$
\frac{1}{p}-\frac{m-j}{N}<0
$$

Hence there is a number $k_{2}>0$ such that

$$
\|u\|_{C^{j}(\bar{G})} \leqq k_{2}\|u\|_{m, p}, \quad \text { for any } u \in W_{p}^{m}(G) .
$$

The imbedding is compact.

(iii) $W_{p}^{m}(G) \subset C^{j, r}(G)$ for any numbers $j, r$ satisfying the relation

$$
\frac{1}{p}-\frac{m-j-r}{N}<0
$$

This imbedding is also compact. 
For the proof of this result we refer to Browder [5] and Nirenberg [28]. For our purposes the following corollary to Sobolev's Imbedding Theorem is particularly important.

Corollary I.4.1. Let $G$ be a bounded domain of class $C^{1}$ in $R^{N}$. Then $W_{p}^{m}(G) \subset L_{r}(G)$ for any $r$ satisfying the relation, $1 \leqq r \leqq N p /(N-m p)$. In this case, there is a constant $k_{3}>0$ such that

$$
\|u\|_{L_{r}(G)} \leqq k_{3}\|u\|_{m, p} \quad \text { for all } u \in W_{p}^{m}(G) .
$$

This imbedding is compact if $1 \leqq r<N p /(N-m p)$. In case $N \leqq m p$, the imbedding is compact for any $r, 1 \leqq r<\infty$.

The following results show how new inequalities can be derived from the properties of imbedding mappings.

LeMmA I.4.2. Let $X_{1}, X_{2}, X_{3}$ be three Banach spaces such that $X_{1} \subset X_{2} \subset X_{3}$. Suppose the imbedding $X_{1} \rightarrow X_{2}$ is compact. Then given any $\varepsilon>0$, there is a $K(\varepsilon)>0$ such that for all $y \in X_{1}$

$$
\|y\|_{x_{2}} \leqq \varepsilon\|y\|_{x_{1}}+K(\varepsilon)\|y\|_{x_{3}} \text {. }
$$

Proof. (Lions [24]). Assume the inequality is false. Thus there is a sequence $\left\{y_{n}\right\}$ in $X_{1}$ such that $\left\|y_{n}\right\|_{x_{2}} \geqq \varepsilon\left\|y_{n}\right\|_{x_{1}}+n\left\|y_{n}\right\|_{x_{3}}$. Setting $v_{n}=y_{n} /\left\|y_{n}\right\|_{x_{1}}$, we obtain

$$
\left\|v_{n}\right\|_{x_{1}}=1 \text { and }\left\|v_{n}\right\|_{x_{2}} \geqq \varepsilon+n\left\|v_{n}\right\|_{x_{3}} .
$$

By the properties of the imbeddings $X_{1} \subset X_{2} \subset X_{3}$, there is a subsequence of $v_{n}$, which we label $v_{n}$, such that $v_{n} \rightarrow v$ strongly in $X_{2}$ and $X_{3}$. On the other hand, using (1) we must have both $\|v\|_{x_{3}}=0$ and $\|v\|_{x_{2}} \geqq \varepsilon>0$, a contradiction.

LEMMA I.4.3. Let $G$ be a bounded domain in $R^{N}$. Then for any $\varepsilon>0$, there is a $K(\varepsilon)>0$, such that for all $u \in \mathscr{W}_{2}^{(m)}(G)$

$$
\|u\|_{L_{2}(G)} \leqq \varepsilon\|u\|_{W_{2}^{m}(G)}^{m}+K(\varepsilon)\|u\|_{L_{1}(G)} .
$$

Proof. As we are considering functions $u(x) \in \mathscr{W}_{2}^{(m)}(G)$, without loss of generality we may assume $G$ is of class $C^{\mathbf{1}}$. Thus by Sobolev's Imbedding Theorem the imbedding $\mathscr{W}_{2}^{(m)}(G) \rightarrow L_{2}(G)$ is compact for any integers $m, N$. Further as $G$ is a bounded domain, $L_{2}(G) \subset L_{1}(G)$. Hence the required result follows from Lemma I.4.2, by setting $X_{1}=\mathscr{W}_{2}^{(m)}(G), X_{2}=L_{2}(G), X_{3}=L_{1}(G)$.

I.5. Elementary facts concerning Banach and Hilbert spaces. Two general notions of convergence will be useful: (1) $y_{n} \rightarrow y$ strongly in a Banach space $X$ if $\left\|y_{n}-y\right\|_{X} \rightarrow 0$ as $n \rightarrow \infty$, and (2) $y_{n} \rightarrow y$ weakly in a Banach space $X$ if $l\left(y_{n}\right) \rightarrow l(y)$ as $n \rightarrow \infty$, for every bounded linear functional $l$ defined on the space $X$. If $y_{n} \rightarrow y$ weakly in a Banach space $X$, and $X \rightarrow X_{1}$ is a compact imbedding, then $y_{n} \rightarrow y$ strongly in the Banach space $X_{1}$. 
We note the following facts concerning weak convergence in the Banach space $X$ : (a) weak limits are unique; (b) if $y_{n} \rightarrow y$ weakly in $X$, then the set of $X$-norms of the sequence $\left\{y_{n}\right\}$ is uniformly bounded; (c) if $X$ is a separable, reflexive Banach space, any set of elements in $X$, with uniformly bounded $X$-norms, is weakly precompact.

(d) Riesz Representation THEOREM. Let $l(y)$ be a bounded linear functional defined on the Hilbert space $H$. Then $l(y)$ has the unique representation $l(y)=(y, z)_{H}$, where $z$ is a fixed element of $H$.

I.6. Operators in Banach and Hilbert spaces. Two types of operators mapping the Banach space $X_{1}$ into the Banach space $X_{2}$ arise in the present work.

(a) Nonlinear composition operators. $B u=f(u)$, where $f(t)$ is a real-valued continuous function on $R^{1}$, and the Banach spaces $X_{1}$ and $X_{2}$ are considered as spaces of real-valued functions.

(b) Linear elliptic differential operators $A$. We shall show that many elliptic differential operators $A$ of order $2 m$ can be uniquely associated with a bounded linear operator $\mathscr{A}$ mapping $\mathscr{W}_{2}^{(m)}(G)$ into itself.

The following standard definitions are convenient. Let $B$ be an operator from the Banach space $X_{1}$ into the Banach space $X_{2}$; then $B$ is continuous if it maps strongly convergent sequences in $X_{1}$ into strongly convergent sequences in $X_{2}$, $B$ is bounded if it maps bounded sets in $X_{1}$ into bounded sets in $X_{2}, B$ is uniformly continuous if for every number $\varepsilon>0$ there is a $\delta>0$ such that $\|y-\bar{y}\|_{x}<\delta$ implies $\|B y-B \bar{y}\|_{x_{1}}<\varepsilon, B$ is compact if $B$ maps every bounded set in $X_{1}$ into a precompact set in $X_{2}$. A functional is an operator, not necessarily linear, defined on a Banach space $X_{1}$ with range in the real numbers $R^{1}$. A functional $F$ is called weakly continuous if $F$ maps weakly convergent sequences in $X_{1}$, into convergent sequences of real numbers.

Let $f(t, x)$ be a real-valued function defined on $R^{1} \times G$, continuous in both the $t$ and $x$ variables. Denote by $B$ the operator defined on the set of real-valued functions $u(x)$ on $G$ by

$$
B u(x)=f(u(x), x) .
$$

We note that $B$ maps measurable functions on $G$ into measurable functions on $G$. Further $B$ maps $C(G)$ into itself. The following result, due to M. Vainberg [34], gives conditions under which $B$ maps $L_{p_{1}}(G)$ into $L_{p_{2}}(G)$ in a continuous and bounded manner, without imposing additional smoothness properties on the function $f(t, x)$.

TheOREM I.6.1 (VAINBERG). Suppose the operator $B(u(x))=f(u(x), x)$ maps the space $L_{p}(G)$ into the space $L_{p_{1}}(G)$. Then the operator $B$ is a continuous and bounded mapping if and only if the function $f(t, x)$ satisfies the growth condition

$$
|f(t, x)| \leqq A+k|t|^{p / p_{1}} \quad \text { for all } u \in G, t \in R^{1},
$$

where $A$ and $k$ are positive constants. 
For the proof of this result we refer to Vainberg's paper [34].

We turn now to the second class of operators considered here, linear elliptic differential operators. The following definitions will be convenient. Let $\mathscr{A}$ be a bounded linear mapping of a real Hilbert space $H$ into itself. $\mathscr{A}$ is self-adjoint if $(\mathscr{A} u, v)_{H}=(u, \mathscr{A} v)_{H}$ for all $u, v \in H . \mathscr{A}$ is semi-bounded if there is a constant $c_{0}$ such that $(\mathscr{A} u, u)_{H} \geqq c_{0}(u, u)_{H}$ for all $u \in H . \mathscr{A}$ is positive definite if $\mathscr{A}$ is semibounded and the constant $c_{0}$ is positive.

We now sketch a well-known procedure which associates a linear elliptic differential operator $A$ uniquely to a bounded linear operator $\mathscr{A}$ in a Hilbert space $H$ (cf. II.3).

Let $A$ be a linear elliptic differential operator of order $2 m$ defined on a bounded domain $G$. Suppose $A$ can be written in the divergence form $A u=\sum_{|\alpha|,|\beta| \leqq m} D^{\alpha}\left(a_{\alpha \beta}(x) D^{\beta} u\right)$ where $a_{\alpha \beta}(x)$ are real-valued uniformly bounded functions defined on $G$. Associated with $A$ is the Dirichlet form $a(u, v)$ defined for all $u, v \in \mathscr{W}_{2}^{(m)}(G)$ by $a(u, v)=\sum_{|\alpha|,|\beta| \leqq m} \int_{G} a_{\alpha \beta}(x) D^{\alpha} u D^{\beta} v$. Using Schwarz's inequality and the uniform boundedness of the coefficients $a_{\alpha \beta}(x)$ we conclude

$$
|a(u, v)| \leqq k\|u\|_{m, 2}\|v\|_{m, 2} \text {. }
$$

Thus $a(u, v)$ is a bilinear bounded functional on $\mathscr{W}_{2}^{(m)}(G)$. Thus by the Riesz representation theorem there is a bounded linear operator $\mathscr{A}$ mapping $\mathscr{W}_{2}^{(m)}(G)$ into itself such that $\langle\mathscr{A} u, v\rangle=a(u, v)$.

EXAMPLES. (a) If $A$ is formally self-adjoint, i.e. $a_{\alpha \beta}(x)=a_{\beta \alpha}(x), \mathscr{A}$ is a selfadjoint operator in $\mathscr{W}_{2}^{m}(G)$. (b) Let $A_{0}=\sum_{|\alpha| \leqq m} D^{\alpha} D^{\alpha}$. Differentiation by parts shows for functions $u$ in $\mathscr{W}_{2}^{m}(G),\left(A_{0} u, u\right)_{L_{2}(G)}=\langle u, u\rangle_{\mathscr{W}_{2}{ }_{G}}^{(m)}$. Thus in the Hilbert space $\mathscr{W}_{2}^{m}(G)$, there is a positive definite operator $\mathscr{A}_{0}$ uniquely associated with $A_{0}$.

The following important result allows us to establish the semi-boundedness of a large class of uniformly elliptic operators.

THEOREM I.6.2 (GÅRDING'S INEQUALITY). Suppose $G$ is a bounded domain in $R^{N}$, and $A$ is a real uniformly elliptic linear differential operator of order $2 m$ with uniformly bounded coefficients and uniformly continuous top order terms; then there are constants $c_{1}>0$ and $c_{2} \geqq 0$ such that for all $u \in \mathscr{W}_{2}^{m}(G)$

$$
a(u, u) \geqq c_{1}\|u\|_{w_{2}^{m}}^{2}-c_{2}\|u\|_{L_{2}(G)}^{2} .
$$

For a proof of this result we refer to the paper of Gårding [13].

II. The eigenvalue problem and its associated operator equation. In this section we state the basic problem of our study and show its equation can be reduced to the solution of an operator equation in the Hilbert space $\mathscr{W}_{2}^{(m)}(G)$.

II.1. The boundary value problem. We shall consider the boundary value problem 


$$
\begin{aligned}
A u-\lambda f(u, x) & =0, \\
\left.u\right|_{\partial G} & =\left.D u\right|_{\partial G}=\cdots=\left.D^{m-1} u\right|_{\partial G}=0,
\end{aligned}
$$

where (a) $G$ is a fixed bounded domain in $R^{N}$, (b) $A$ is a formally self-adjoint uniformly elliptic real linear operator of order $2 m$ with uniformly bounded measurable coefficients and top order terms uniformly continuous ( $A$ is assumed to be given in the divergence form $\left.A u=\sum_{|\alpha|,|\beta| \leqq m} D^{\alpha}\left(a_{\alpha \beta}(x) D^{\beta} u\right)\right)$, (c) $\lambda$ is a real number, and (d) $f(t, x)$ is a real-valued function defined on $R^{1} \times G$, jointly continuous in the $t$ and $x$ variables, with the following properties:

1. $f(0, x) \equiv 0$.

2. $t f(t, x)>0(t \neq 0)$ for all $x \in G$.

3. $f(-t, x)=-f(t, x)$.

4. $f(t, x)$ is a nondecreasing function of $t$ for fixed $x$.

5. For some fixed $x_{1} \in G$ and some positive constant $k$ and all $x \in G$, $f(t, x) \geqq k f\left(t, x_{1}\right)>0$ for $t>0$.

6. (Polynomial growth condition.) $|f(t, x)| \leqq a+k_{1}|t|^{\rho}$ for all $(t, x) \in R^{1} \times G$, where $a, k_{1}$ are nonnegative constants and $\rho=\rho(m, N)$, i.e. $\rho$ depends on the order of the operator $A$ and the dimension of the Euclidean space $R^{N}$ containing G.

If $f(t, x)$ is analytic in $t, f(t, x)$, for each $x$, reduces to a polynomial in $t$ with terms of odd degree and coefficients $b_{k}(x)$, i.e. $f(t, x)=\sum_{k=0}^{l} b_{k}(x) t^{2 k+1}$ where $(2 l+1) \leqq \rho(m, N)$. We shall call the family of functions $f(t, x)$ which satisfy all conditions of II.1 (d) $\mathscr{F}(\rho)$, where this family is indexed by the exponent $\rho$ occuring in the polynomial growth condition.

In many special circumstances the conditions defining $\mathscr{F}(\rho)$ can be greatly reduced; we shall make special note of such circumstances as they arise. We note that property 4 is motivated by physical considerations (cf. Duff [11]).

II.2. Classical and generalized eigenfunctions. Recent studies of nonlinear elliptic partial differential equations (cf. Browder [7], [8] and Ladyzenskaya [22]) have shown the advantage to be gained by broadening the meaning of equation (II.1.1) to allow solutions that are not necessarily smooth enough to satisfy (II.1.1) in an exact sense. Thus we make the following definitions.

DEFINITION II.2.1. A classical solution of the boundary value problem (II.1.1) is a function $u(x)$ with the following properties: (1) $u(x) \in C^{2 m}(G),(2) u(x) \in C^{m-1}(\bar{G})$, (3) $u(x)$ satisfies the equation $A u-\lambda f(u, x)=0$ in $G$, as well as the boundary values $\left.u(x)\right|_{\partial G}=\left.D u(x)\right|_{\partial G}=\cdots=\left.D^{m-1} u(x)\right|_{\partial G}=0$ at each point $x$ of $\partial G$.

DEFINITION II.2.2. A generalized solution of the boundary value problem (II.1.1) is a function $u(x)$ with the following properties: (1) $u(x) \in \mathscr{W}_{2}^{(m)}(G)$ and (2) $\sum_{|\alpha|,|\beta| \leqq m} \int_{G} a_{\alpha \beta}(x) D^{\alpha} u D^{\beta} v=\lambda \int_{G} f(u, x) v$, for all functions $v(x) \in \mathscr{W}_{2}^{(m)}(G)$.

Differentiation by parts shows that every classical solution of (II.1.1) is a generalized solution. The converse is, in general, not true. The following lemma gives a partial converse. 
LEMMA II.2.1. Suppose $u(x)$ is any generalized solution of the boundary value problem (II.1.1). Suppose in addition that $u(x) \in C^{m-1}(\bar{G}) \cap C^{2 m}(G)$ and $G$ is of class $C^{m}$; then $u(x)$ is a classical solution of (II.1.1).

We postpone the proof of this lemma to $\S \mathrm{V}$, where the discussion of the regularity of generalized solutions is systematically investigated.

The boundary value problem (II.1.1) always has a classical solution, namely the trivial one, $u(x) \equiv 0$ in $G$. The following definitions focus attention on nontrivial solutions.

Definition II.2.3. A classical eigenfunction for (II.1.1) is a classical solution of (II.1.1) such that $u(x) \neq 0$ in $G$.

Definition II.2.4. A generalized eigenfunction for (II.1.1) is a generalized solution of (II.1.1) such that $u(x) \not \equiv 0$, apart from a set of measure 0 , in $G$.

II.3. The operator equation. A special feature of generalized solutions is the fact that they are often expressible as solutions of an operator equation in a Hilbert space, or more generally, in a reflexive, separable Banach space. Indeed, the following result holds in the present situation.

THEOREM II.3.1. Suppose the function $f(t, x)$ is a member of the class $\mathscr{F}(\rho)$, where

$$
\begin{aligned}
& 0 \leqq \rho<\frac{N+2 m}{N-2 m}, \quad N>2 m, \\
& 0 \leqq \rho<\infty, \quad N \leqq 2 m .
\end{aligned}
$$

Then the generalized solutions of the boundary value problem(II.1.1)are identical with the solutions of the operator equation $\mathfrak{A} u-\lambda B u=0$ defined on the Hilbert space $\mathscr{W}_{2}^{(m)}(G)$, where $\mathfrak{U}$ is a self-adjoint bounded linear operator mapping $\mathscr{W}_{2}^{(m)}(G)$ into itself and $B$ is a continuous, compact, not necessarily linear, mapping of $\mathscr{W}_{2}^{(m)}(G)$ into itself with $B(0)=0$.

The proof of this theorem is based on the following two lemmas.

LEMMA II.3.2. Let $A$ be an elliptic differential operator of order $2 m$ defined on $G$ with the properties described in II.1 (b). Then for any $u, v \in \mathscr{W}_{2}^{(m)}(G)$, $a(u, v)=\langle\mathfrak{A} u, v\rangle_{m, 2}$ (cf. I.3) where $\mathfrak{A}$ is a self-adjoint bounded linear mapping of $\mathscr{W}_{2}^{(m)}(G)$ into itself. Further there are constants $c_{1}>0$ and $c_{2} \geqq 0$ such that for all $u \in \mathscr{W}_{2}^{(m)}(G)$

$$
\langle\mathfrak{A} u, u\rangle_{m, 2} \geqq c_{1}\|u\|_{m, 2}^{2}-c_{2}\|u\|_{0,2}^{2} \text {. }
$$

Proof. We write $a(u, v)=\sum_{|\alpha|,|\beta| \leqq m} \int_{G} a_{\alpha \beta}(x) D^{\alpha} u D^{\beta} v$. Let $M$ be the uniform bound of the coefficients $a_{\alpha \beta}(x)$. Hence by Schwarz's inequality

$$
|a(u, v)| \leqq M\|u\|_{m, 2}\|v\|_{m, 2} .
$$

Thus, for any $u, v \in \mathscr{W}_{2}^{(m)}(G), a(u, v)$ defines a bounded bilinear functional 
on the Hilbert space $\mathscr{W}_{2}^{(m)}(G)$. Thus by the Riesz representation theorem, there is a uniquely defined bounded linear operator $\mathfrak{A}$ mapping $\mathscr{W}_{2}^{(m)}(G)$ into itself and such that $a(u, v)=\langle\mathfrak{U} u, v\rangle_{m, 2}$. As $A$ is formally self-adjoint, $a(u, v)$ is symmetric and as $a(u, v)=\langle\mathfrak{A} u, v\rangle_{m, 2}, \mathfrak{A}$ is self-adjoint. The final inequality is an immediate consequence of Gårding's inequality, Theorem I.6.2.

Lemma II.3.3. Suppose the function $f(t, x)$ is a member of the class $\mathscr{F}(\rho)$, where

$$
\begin{array}{ll}
0 \leqq \rho<\frac{N+2 m}{N-2 m} & \text { if } N>2 m, \\
0 \leqq \rho<\infty & \text { if } N \leqq 2 m .
\end{array}
$$

Then $\int_{G} f(u, x) v=\langle B u, v\rangle_{m, 2}$ for any $u, v \in \mathscr{W}_{2}^{(m)}(G)$, where $B$ is a compact, continuuous, not necessarily linear, mapping of $\mathscr{W}_{2}^{(m)}(G)$ into itself. Further, the form $\langle B u, v\rangle_{m, 2}$ is a weakly continuous function of the elements $u, v$.

Proof. As $f(t, x)$ is continuous on $R^{1} \times G$ for any $u, v \in \mathscr{W}_{2}^{(m)}(G), f(u, x) v$ is a measurable function on $G$. To show $f(u, x) v$ is integrable over the bounded domain $G$, it is necessary to show that it is bounded in absolute value by an integrable function. Indeed by the polynomial growth condition on $f(t, x)$, the function $|f(u, x) v|$ is dominated by the function $a|v|+k_{1}|u|^{\rho}|v|$ a.e. in $G$, and, as $G$ is a bounded domain,

$$
\int_{G}\left(a|v|+k_{1}|u|^{\rho}|v|\right) \leqq a\|v\|_{L_{1}(G)}+k_{1}\left\|u^{\rho}\right\|_{L_{r^{\prime}(G)}}\|v\|_{L_{r(G)}}
$$

for suitable $r$ and $r^{\prime}$. To insure this latter expression is finite we must choose $r$ and $\rho$ in accordance with the corollary of Sobolev's Imbedding Theorem. It suffices to choose $\rho<(N+2 m) /(N-2 m)$ if $N>2 m$ or any positive finite number if $N \leqq 2 m$. In this case, (1) is finite by Hölder's inequality, for then $1<r$ $<2 N /(N-2 m)$ if $N>2 m$ and $1<r<\infty$ otherwise.

Now $\int_{G} f(u, x) v$ defines a linear functional on $\mathscr{W}_{2}^{(m)}(G)$ in $v$, which we denote by $I(v)$. Using the polynomial growth condition on $f(t, x)$ and Hölder's inequality, we write, by virtue of Sobolev's Imbedding Theorem $|I(v)| \leqq g\left(\|u\|_{m, 2}\right)\|v\|_{m, 2}$ where $g(r)$ is a continuous monotone increasing function of the nonnegative variable $r$. Thus $I(v)$ defines a bounded linear functional on the Hilbert space $\mathscr{W}_{2}^{(m)}(G)$ and by the Riesz representation theorem $I(v)=\langle z, v\rangle_{m, 2}$ for some fixed $z \in \mathscr{W}_{2}^{(m)}(G)$. We now define an operator $B$ as follows:

$$
B u=z \text {, where } I(v)=\int_{G} f(u, x) v=\langle z, v\rangle_{m, 2} .
$$

Thus $B$ is a well-defined mapping of $\mathscr{W}_{2}^{(m)}(G)$ into itself.

We proceed now to show that $B$ is a continuous, compact operator. Since $\mathscr{W}_{2}^{(m)}(G)$ is a Hilbert space, it suffices to show that $B$ maps weakly convergent 
sequences in $\mathscr{W}_{2}^{(m)}(G)$ into strongly convergent sequences in $\mathscr{W}_{2}^{(m)}(G)$. To this end, let $u_{n} \rightarrow u$ weakly in $\mathscr{W}_{2}^{(m)}(G)$; hence $u \in \mathscr{W}_{2}^{(m)}(G)$ and

$$
\begin{aligned}
\left\|B u_{n}-B u\right\|_{m, 2} & =\sup _{\|v\|_{m}, 2 \leqq 1}\left\langle B u_{n}-B u, v\right\rangle_{m, 2} \\
& =\sup _{\|v\|_{m}, 2 \leqq 1} \int_{G}\left(f\left(u_{n}, x\right)-f(u, x)\right) v .
\end{aligned}
$$

We show this latter expression tends to 0 as $n \rightarrow \infty$.

Case I $(N>2 m)$. Using the corollary of Sobolev's Imbedding Theorem and Hölder's inequality with $p=2 N /(N+2 m)$ and $q=2 N /(N-2 m)$, we obtain from (2)

$$
\sup _{\|v\|_{m, 2} \leqq 1} \int_{G}\left(f\left(u_{n}, x\right)-f(u, x)\right) v \leqq K\left\|f\left(u_{n}, x\right)-f(u, x)\right\|_{L_{2 N /(N+2 m)}}
$$

where $K$ is a positive constant independent of $v \in \mathscr{W}_{2}^{(m)}(G)$. By the hypothesis of the lemma there is an $\varepsilon>0$ such that $f(t, x) \in \mathscr{F}(\rho)$ and $(1+\varepsilon) \rho=(N+2 m) /(N-2 m)$. Set $(1+\varepsilon) s=2 N /(N-2 m)$. Then by Sobolev's Imbedding Theorem the imbedding $i: W_{2}^{m}(G) \rightarrow L_{s}$ is compact and by Vainberg's theorem the mapping $\tilde{f:} L_{s} \rightarrow L_{2 N /(N+2 m)}$ is continuous, where $\bar{f}(u)=f[u(x), x]$. Thus the mapping fi: $W_{2}^{m}(G) \rightarrow L_{2 N /(N+2 m)}$ is continuous and compact. As $u_{n} \rightarrow u$ weakly in $\mathscr{W}_{2}^{(m)}(G)$ the expression on the right-hand side of (3) tends to 0 , as $n \rightarrow \infty$, as required.

Case II $(N \leqq 2 m)$. We follow the same procedure as in Case I. Using the corollary of Sobolev's Imbedding Theorem and Hölder's inequality with any $p$, $1<p<\infty$, and $1 / q=1-1 / p$, we obtain from (2)

$$
\sup _{\|v\|_{m}, 2 \leqq 1} \int_{G}\left[f\left(u_{n}, x\right)-f(u, x)\right] v \leqq K_{1}\left\|f\left(u_{n}, x\right)-f(u, x)\right\|_{L_{p}}
$$

where $K$ is a constant independent of $v \in \mathscr{W}_{2}^{(m)}(G)$. By the hypothesis of the lemma, $f(t, x)$ is in some class $\mathscr{F}(\rho), 0<\rho<\infty$. Choose $p$ so that $\rho p>1$. Thus as in Case I the mapping $\bar{f} i: W_{2}^{m}(G) \rightarrow L_{\rho p}(G) \rightarrow L_{p}(G)$ is continuous and compact. Hence the expression on the right-hand side of (4) tends to 0 , as $n \rightarrow \infty$, as required.

To complete the lemma we show $\langle B u, v\rangle_{m, 2}$ is a weakly continuous function of the elements $u, v \in \mathscr{W}_{2}^{(m)}(G)$. Let $u_{n} \rightarrow u, v_{n} \rightarrow v$ weakly in $\mathscr{W}_{2}^{(m)}(G)$. Then by Schwarz's inequality

$$
\begin{aligned}
\left|\left\langle B u_{n}, v_{n}\right\rangle_{m, 2}-\langle B u, v\rangle_{m, 2}\right| & =\left|\left\langle B u_{n}-B u, v_{n}\right\rangle_{m, 2}-\left\langle B u, v-v_{n}\right\rangle_{m, 2}\right| \\
& \leqq\left\|v_{n}\right\|_{m, 2}\left\|B u_{n}-B u\right\|_{m, 2}+\left|\left\langle B u, v-v_{n}\right\rangle_{m, 2}\right|
\end{aligned}
$$

As $v_{n} \rightarrow v$ weakly in $\mathscr{W}_{2}^{(m)}(G)$, and $B$ maps weakly convergent sequences into strongly convergent sequences, this latter expression tends to 0 as $n \rightarrow \infty$, as required.

Proof of Theorem II.3.1. Let $u(x)$ be any generalized solution of the boundary 
value problem (II.1.1); then $a(u, v)-\lambda \int_{G} f(u, x) v=0$ for any $v \in \mathscr{W}_{2}^{(m)}(G)$. Using Lemmas II.3.2 and II.3.3 and the hypothesis of the theorem we conclude

$$
\langle\mathfrak{A} u-\lambda B u, v\rangle_{m, 2}=0 \text { for all } v \in \mathscr{W}_{2}^{(m)}(G) .
$$

As $\mathscr{W}_{2}^{(m)}(G)$ is a Hilbert space, $\mathfrak{A} u-\lambda B u=0$. Conversely if $\mathfrak{A} u-\lambda B u=0$, $\langle\mathfrak{A} u-\lambda B u, v\rangle_{m, 2}=0$ for all $v \in \mathscr{W}_{2}^{(m)}(G)$. Hence using the linearity of the inner product and the lemmas of this subsection $a(u, v)-\lambda \int_{G} f(u, x) v=0$, as required. Also $\langle B(0), v\rangle_{m, 2}=\int_{G} f(0, x) v \equiv 0$; therefore $B(0)=0$.

III. Energy levels in the Hilbert space $\mathscr{W}_{2}^{(m)}(G)$. In this section we define and investigate certain sets of functions in the Hilbert space $\mathscr{W}_{2}^{(m)}(G)$. We refer to these sets as "energy levels." Their properties will be of basic importance in the remainder of this work.

III.1. Properties of the function $F(t, x)$. Let $f(t, x)$ be a real-valued continuous function defined on $R^{1} \times G$, of class $\mathscr{F}(\rho)$. For purposes of reference we repeat: 1. $f(0, x) \equiv 0$. 2. $t f(t, x)>0(t \neq 0)$ for all $x \in G$. 3. $f(-t, x)=-f(t, x)$. 4. $f(t, x)$ is a nondecreasing function of $t$ for fixed $x$. 5. For some fixed $x_{1} \in G$, and some constant $k, f(t, x) \geqq k f\left(t, x_{1}\right)>0$ for $t>0$. 6. (Polynomial growth condition.) $|f(t, x)| \leqq a+k_{1}|t|^{\rho}$ for all $(t, x) \in R^{1} \times G$, where $a, k_{1}, \rho$ are nonnegative constants.

DeFinITION III.1.1. $\boldsymbol{F}(t, x) \equiv \int_{0}^{t} f(s, x) d s$.

In the next lemma, we state the properties of the function $F(t, x)$, regarding $t$ as variable and $x$ in $G$ fixed.

LEMMA III.1.1. For fixed $x \in G, F(t, x)$ is a nonnegative convex function of class $C^{1}$. For $t \geqq 0 F(t, x)$ is a monotone strictly increasing function of $t$, with $F(0, x)=0, F(\infty, x)=\infty$.

Proof. As $f(t, x)$ is an odd continuous function of $t, F(t, x)$ is nonnegative and of class $C^{1}$, due to the integral representation, $F(t, x)=\int_{0}^{t} f(s, x) d s$. The integral representation also shows $F(0, x) \equiv 0$ and $F(\infty, x)=\infty$. In addition as $f(t, x)$ is nondecreasing in $t, F(t, x)$ is a strictly monotone increasing function in $t$. To prove $F(t, x)$ is convex in $t$, it suffices to prove

$$
F\left(\frac{t_{1}+t_{2}}{2}, x\right) \leqq \frac{1}{2}\left\{F\left(t_{1}, x\right)+F\left(t_{2}, x\right)\right\} \text { for } t_{1}, t_{2} \in R^{1} .
$$

To this end we first assume $0 \leqq t_{1} \leqq t_{2}$; then $F\left(t_{2}, x\right)=\int_{0}^{\left(t_{1}+t_{2}\right) / 2}+\int_{\left(t_{1}+t_{2}\right) / 2}^{t_{2}}$ and $F\left(t_{1}, x\right)=\int_{0}^{\left(t_{1}+t_{2}\right) / 2}-\int_{t_{1}}^{\left(t_{1}+t_{2}\right) / 2}$. As $f(t, x)$ is nondecreasing, $\int_{\left(t_{1}+t_{2}\right) / 2}^{t_{2}} f(s, x) d s$ $\geqq \int_{t_{1}}^{\left(t_{1}+t_{2}\right) / 2} f(s, x) d s$. Thus $F\left(t_{1}, x\right)+F\left(t_{2}, x\right) \geqq 2 \int_{0}^{\left(t_{1}+t_{2}\right) / 2} f(s, x) d s=2 F\left(\left(t_{1}+t_{2}\right) / 2, x\right)$. For arbitrary $t_{1}, t_{2}$, we note $F(t, x)=F(|t|, x)$. Thus $F\left(\left(t_{1}+t_{2}\right) / 2, x\right)$ $=F\left(\left|t_{1}+t_{2}\right| / 2, x\right) \leqq F\left(\left(\left|t_{1}\right|+\left|t_{2}\right|\right) / 2, x\right) \leqq \frac{1}{2}\left(F\left(\left|t_{1}\right|, x\right)+F\left(\left|t_{2}\right|, x\right)\right)$ $\leqq \frac{1}{2}\left\{F\left(t_{1}, x\right)+F\left(t_{2}, x\right)\right\}$. 
LEMMA III.1.2. The function $F(t, x)$ has the following properties: (1) $F\left(t_{1}, x\right)-F\left(t_{2}, x\right)=\left(t_{2}-t_{1}\right) \int_{0}^{1} f\left(t_{1}+s\left(t_{2}-t_{1}\right), x\right) d s$, (2) $F(t, x)=t \int_{0}^{1} f(t s, x) d s$, (3) there are positive constants $c, d$ such that for all $t \in R^{1}$ and $x$ in $G$ fixed,

$$
c t f(t, x) \leqq F(t, x) \leqq d t f(t, x) .
$$

Proof. By the definition of $F(t, x), F\left(t_{2}, x\right)-F\left(t_{1}, x\right)=\int_{t_{1}}^{t_{2}} f\left(s^{\prime}, x\right) d s^{\prime}$. We obtain (1) by making the substitution $s^{\prime}=\left(t_{2}-t_{1}\right) s+t_{1}$ in the last integral. We obtain (2) by setting $t_{1}=0$ and $t_{2}=t$ in (1), recalling $F(0, x) \equiv 0$. The fact that $F(t, x) \leqq d t f(t, x)$ is an immediate consequence of the fact that $f(t, x)$ is a continuous odd and nondecreasing function in $t$ for fixed $x$. The fact that $c t f(t, x) \leqq F(t, x)$ is an immediate consequence of the polynomial growth condition on $f(t, x)$.

III.2. Elementary properties of energy levels. We proceed now to describe the energy levels $\partial M_{R}$ associated with the function $F(t, x)$ and the boundary value problem (II.1.1). Throughout the remainder of this section we shall assume that $f(t, x) \in \mathscr{F}(\rho)$ with $0 \leqq \rho<(N+2 m) /(N-2 m)$ if $N>2 m$, or $0 \leqq \rho<\infty$ otherwise. Let $R$ be any fixed real number $0<R<\infty$.

DEFINITION III.2.1. $M_{R}$ is the set of all functions $u(x)$ such that $(1) u(x) \in \mathscr{W}_{2}^{(m)}(G)$ and (2) $\int_{G} F(u(x), x) \leqq R$.

DEFINITION III.2.2. $\partial M_{R}$ is the set of all functions $u(x)$ such that $(1) u(x) \in \mathscr{W}_{2}^{(m)}(G)$ and (2) $\int_{G} F(u(x), x)=R$.

EXAMPLE. Suppose $f(t, x)=2 t$; then $F(t, x)=t^{2}$. Thus $M_{R}=\left\{u \mid u \in \mathscr{W}_{2}^{(m)}(G)\right.$, $\left.\|u\|_{L_{2}(G)}^{2} \leqq R\right\}$, and $\partial M_{R}=\left\{u \mid u \in \mathscr{W}_{2}^{(m)}(G),\|u\|_{L_{2}(G)}^{2}=R\right\}$. Clearly, in this example, $M_{R}$ is a well-defined closed convex set in $\mathscr{W}_{2}^{(m)}(G)$. As the imbedding $\mathscr{W}_{2}^{(m)}(G) \rightarrow L_{2}(G)$ is compact, $\partial M_{R}$ is weakly closed.

We proceed now to extend the properties of the above example to the general sets $M_{R}$ and $\partial M_{R}$.

LEMMA III.2.1 (GEOMETRY OF ENERGY LEVELS). Let $R$ be a fixed positive number. Then the following properties hold:

(1) $M_{R}$ contains nonzero elements of $\mathscr{W}_{2}^{(m)}(G)$.

(2) $M_{R}$ is a closed, convex set in $\mathscr{W}_{2}^{(m)}(G)$.

(3) $\partial M_{R}$ is symmetric about the origin.

(4) On $\partial M_{R},\|u\|_{W_{2}(G)}^{m} \geqq c(R)>0$ for some constant $c(R)$, independent of $u$.

(5) In general, $M_{R}$ is an unbounded set in $\mathscr{W}_{2}^{(m)}(G)$.

Proof. (1) Given an element $u \in \mathscr{W}_{2}^{(m)}(G)$ we must show that $\int_{G} F(u, x)$ has a well-defined numerical value. As $F(t, x)$ is continuous in $t$ and $x$ the function $F(u(x), x)$ is measurable. To show $F(u(x), x)$ is integrable over $G$, we show that it can be dominated by an integrable function over G. By Lemma III.1.2 (3) and the polynomial growth condition on $f(t, x): F(t, x) \leqq k_{1}|t|+k_{2}|t|^{\rho+1}$. Hence using Sobolev's Imbedding Theorem and the fact $f(t, x) \in \mathscr{F}(\rho)$, 


$$
\begin{aligned}
\int_{G}\left(K_{1}|u|+K_{2}|u|^{\rho+1}\right) & =K_{1}\|u\|_{L_{1}}+K_{2}\|u\|_{L_{\rho+1}+1}^{\rho+1} \\
& \leqq K_{3}\|u\|_{m, 2}<\infty
\end{aligned}
$$

Thus $M_{R}$ contains nonzero elements of $\mathscr{W}_{2}^{(m)}(G)$.

(2) To show $M_{R}$ is a convex set in $\mathscr{W}_{2}^{(m)}(G)$, we consider any two elements $u_{1}, u_{2} \in \partial M_{R}$. Then due to the convexity of $F(t, x)$ in $t$, for any $t, 0 \leqq t \leqq 1$, $\int_{G} F\left(t u_{1}+(1-t) u_{2}, x\right) \leqq t \int_{G} F\left(u_{1}, x\right)+(1-t) \int_{G} F\left(u_{2}, x\right) \leqq R$.

The demonstration of the fact that $M_{R}$ is a closed set in $\mathscr{W}_{2}^{(m)}(G)$ is a prelude to the more subtle result of Lemma III.3.3. We shall show that $\int_{G} F(u, x)$ is a continuous functional on $\mathscr{W}_{2}^{(m)}(G)$. Let $\left\{u_{n}\right\}$ be a strongly convergent sequence in $\mathscr{W}_{2}^{(m)}(G)$ converging to $u$. Then using Lemma III.1.2 and Fubini's theorem we can write:

$$
\begin{aligned}
\int_{G}\left(F\left(u_{n}, x\right)-F(u, x)\right) & =\int_{G}\left(u_{n}-u\right) \int_{0}^{1} f\left[u+s\left(u_{n}-u\right)\right] d s \\
& =\int_{0}^{1} d s \int_{G}\left(u_{n}-u\right) f\left[u+s\left(u_{n}-u\right)\right] .
\end{aligned}
$$

Now for fixed $s, 0 \leqq s \leqq 1$, by Lemma II.3.3,

$$
\int_{G}\left(u_{n}-u\right) f\left[u+s\left(u_{n}-u\right)\right]=\left\langle u_{n}-u, B\left[u+s\left(u_{n}-u\right)\right]\right\rangle_{m, 2} .
$$

As $B$ is a compact operator and the elements $\left\{u+s\left(u_{n}-u\right)\right\}$ are uniformly bounded in $\mathscr{W}_{2}^{(m)}(G)$, the set of elements $\left\{B\left(u+s\left(u_{n}-u\right)\right)\right\}$ is again uniformly bounded in $\mathscr{W}_{2}^{(m)}(G)$, by $M$, say. Thus by Schwarz's inequality

$$
\left\langle u_{n}-u, B\left[u+s\left(u_{n}-u\right)\right]\right\rangle_{m, 2} \leqq M\left\|u_{n}-u\right\|_{m, 2} \text {. }
$$

As $u_{n} \rightarrow u$ strongly in $\mathscr{W}_{2}^{(m)}(G)$ we conclude that

$$
\int_{G}\left(u_{n}-u\right) f\left[u+s\left(u_{n}-u\right)\right] \rightarrow 0 \text { as } n \rightarrow \infty .
$$

Further by the polynomial growth condition on $f$ and Sobolev's Imbedding Theorem $\int_{G}\left(u_{n}-u\right) f\left[u+s\left(u_{n}-u\right)\right]$ is dominated by a polynomial in $s$ with uniformly bounded coefficients. Thus by the Lebesgue Dominated Convergence Theorem, $\int_{G} F\left(u_{n}, x\right) \rightarrow \int_{G} F(u, x)$.

Thus $u_{n} \in M_{R}$ implies $u \in M_{R}$, i.e. $M_{R}$ is closed.

(3) As $F(t, x)=F(-t, x), \int_{G} F(u, x)=\int_{G} F(-u, x)$. Thus $M_{R}$ is symmetric about the origin.

(4) The fact that the elements $u$ of $\partial M_{R}$ have $\|u\|_{m, 2} \geqq c_{R}>0$ is a consequence of the inequality associated with Sobolev's Imbedding Theorem. Indeed, let $u$ be an arbitrary element of $\partial M_{R}$. Then by the argument used in part (1) of this lemma $R=\int_{G} F(u, x) \leqq K_{3}\|u\|_{m, 2}^{r}$, for $r>0$. Thus $\|u\|_{m, 2} \geqq c_{R}>0$. 
(5) To show $M_{R}$ is, in general, unbounded in $\mathscr{W}_{2}^{(m)}(G)$, we consider the special case: $m=1, f(t, x)=2 t, F(t, x)=t^{2}$. Thus the set $M_{1}=\left\{u \mid u \in \mathscr{W}_{2}^{(1)}(G), \int_{G} u^{2} \leqq 1\right\}$. Let $G=(-\varepsilon, 1+\varepsilon)$ for some $\varepsilon>0$. To obtain our result we need only produce a set of functions in $\mathscr{W}_{2}^{(1)}(G)$ with values in $[0,1]$, but with rapidly increasing oscillation. For example,

$$
\begin{aligned}
& u_{1}(x)= \begin{cases}2 x, & 0 \leqq x<\frac{1}{2}, \\
2-2 x, & \frac{1}{2} \leqq x \leqq 1, \\
0, & \text { otherwise }\end{cases} \\
& u_{n}(x)= \begin{cases}u_{n-1}(2 x), & 0 \leqq x<\frac{1}{2} \\
u_{n-1}(2 x-1), & \frac{1}{2} \leqq x \leqq 1 \\
0, & \text { otherwise. }\end{cases}
\end{aligned}
$$

Here $\left\|u_{n}\right\|_{L_{2}(G)}^{2} \leqq 1$, but $\left\|u_{n}\right\|_{1,2} \geqq 2^{n}$. (By using polar coordinates, we can extend this example to any bounded domain $G$ in $R^{N}$.)

LEMMA III.2.2 (HOMOGENEITY PROPERTY OF $\partial M_{R}$ ). Let $u \varepsilon \partial M_{R}$ be given, suppose $R_{1}>R>R_{2}>0$; then there are uniquely defined numbers $b_{1}>1>b_{2}>0$ such that (1) $b_{1} u \in \partial M_{R_{1}}$ and (2) $b_{2} u \in \partial M_{R_{3}}$.

Proof. We fix $u \in \partial M_{R}$, and denote $\mathscr{I}(b)=\int_{G} F(b u, x)$ for $b \geqq 0$. We obtain our conclusion by showing $\mathscr{I}(b)$ is a continuous, monotone strictly increasing function of $b$ with $\mathscr{I}(0)=0$ and $\mathscr{I}(\infty)=\infty$. $\mathscr{I}(b)$ is a continuous function of $b$ as $\int_{G} F(u, x)$ is a continuous functional on $\mathscr{W}_{2}^{(m)}(G)$. To show $\mathscr{I}(b)$ is strictly monotone increasing, suppose $b_{1}>b>0$. Then by Lemma III.1.2 and Fubini's theorem

$$
\begin{aligned}
& \mathscr{I}\left(b_{1}\right)-\mathscr{I}(b)=\int_{G}\left\{F\left(b_{1} u, x\right)-F(b u, x)\right\} \\
&=\int_{0}^{1} d s \int_{G}\left(b_{1}-b\right) u f\left[\left\{b+s\left(b_{1}-b\right)\right\} u, x\right]>0, \\
& \text { as } t f(t, x)>0 \quad(t \neq 0) .
\end{aligned}
$$

As $F(0, x) \equiv 0, \mathscr{I}(0)=0$. Now let $n>1$; then $\mathscr{I}(n)=\int_{G} F(n u, x)$. Using Lemma III.1.2 and Fubini's theorem, $\mathscr{I}(n)=n \int_{0}^{1} d s \int_{G} u f(n s u, x) \geqq n \mathscr{I}(1)$.

Hence, as $n \rightarrow \infty, \mathscr{I}(n) \rightarrow \infty$.

III.3. Subtler properties of energy levels.

LEMMA III.3.1 ( $L_{1}$-BOUNDEDNESS LEMMA). Let $R$ be a fixed positive number. Then, on $\partial M_{R},\|u\|_{L_{1}(G)} \leqq g(R)$, where $g(R)$ is a monotone increasing function of $R$, independent of $u$.

Proof. Using property (5) of $f(t, x)$, we note $F\left(t, x_{1}\right)$ is a convex function of $t$ 
and $F(t, x) \geqq K F\left(t, x_{1}\right)$, for some constant $K$ independent of $t$. Thus by Jensen's integral inequality for convex functions (cf. Krasnoselskiī and Rutitskī [20])

$$
\begin{aligned}
& F\left\{\frac{\int_{G}|u(x)|}{\text { meas } G}, x_{1}\right\} \leqq \frac{\int_{G} F\left(|u(x)|, x_{1}\right)}{\text { meas } G} \\
& \leqq \frac{\int_{G} F\left(u(x), x_{1}\right)}{\text { meas } G} \\
& \leqq \frac{K \int_{G} F(u(x), x)}{\text { meas } G} \\
& \leqq \frac{K R}{\text { meas } G} \quad \text { (as } u \in \partial M_{R} \text { ). }
\end{aligned}
$$

As $F\left(t, x_{1}\right)$ is a strictly increasing monotone function of $t, F\left(t, x_{1}\right)$ has a monotone strictly increasing inverse function, which we denote by $\bar{g}(t)$. Hence

$$
\int_{G}|u(x)| \leqq \text { meas } G \bar{g}\left(\frac{k R}{\text { meas } G}\right)
$$

i.e. $\|u\|_{L_{1}(G)} \leqq g(R)$, when $g(R)$ denotes the above constant.

LEMMA III.3.2. $\int_{G} F(u, v)$ defines a weakly continuous functional on $\mathscr{W}_{2}^{(m)}(G)$.

Proof. Let $u_{n} \rightarrow u$ weakly in $\mathscr{W}_{2}^{(m)}(G)$. Thus the set $\left\{u_{n}\right\}$ is uniformly bounded in $\mathscr{W}_{2}^{(m)}(G)$, and by the argument of Lemma III.2.1 (2) it is sufficient to prove $\int_{G}\left(u_{n}-u\right) f\left[u+s\left(u_{n}-u\right)\right] \rightarrow 0$ for fixed $s, 0 \leqq s \leqq 1$. By Lemma II.3.3 this latter expression can be written $\left\langle B\left[u+s\left(u_{n}-u\right)\right], u_{n}-u\right\rangle_{m, 2}$. As $\langle B u, v\rangle_{m, 2}$ is a weakly continuous function of $u$ and $v,\left\langle B\left[u+s\left(u_{n}-u\right)\right], u_{n}-u\right\rangle_{m, 2}$ $\rightarrow\langle B u, 0\rangle_{m, 2}=0$, as required.

LEMmA III.3.3. Let $R$ be a fixed positive number. Then $\partial M_{R}$ is weakly closed.

Proof. Let $\left\{u_{n}\right\}$ be a weakly convergent sequence of elements of $\partial M_{R}$. Denote the weak limit of $\left\{u_{n}\right\}$ by $u$. By Lemma III.3.2, $\int_{G} F\left(u_{n}, x\right) \rightarrow \int_{G} F(u, x)$. As $u_{n} \in \partial M_{R}, \int_{G} F\left(u_{n}, x\right)=R$. Thus $\int_{G} F(u, x)=R$ and $u \in \partial M_{R}$.

LEMMA III.3.4 (VARIATIONAL LEMMA). Let $\left[u_{n}\right]$ be any sequence of elements selected from $\partial M_{R}$, for fixed $R$, with uniformly bounded $\mathscr{W}_{2}^{(m)}(G)$ norms. Let $\pi$ be an arbitrary fixed element of $\mathscr{W}_{2}^{(m)}(G)$. Then given any $\varepsilon>0$ there is a number $h_{\varepsilon}>0$, depending on $\varepsilon$, such that for each $h,|h|<h_{\varepsilon}$, there is a sequence of real numbers $\left\{\sigma_{n}\right\}$ with the properties:

(1) $\left\{u_{n}+h\left(\sigma_{n} u_{n}+\pi\right)\right\} \in \partial M_{R}$.

(2) $\left|\sigma_{n}+\left\langle B u_{n}, \pi\right\rangle_{m, 2}\right|\left\langle B u_{n}, u_{n}\right\rangle_{m, 2} \mid \leqq R \varepsilon$, where $R$ is a positive constant independent of $\varepsilon$ and $n$.

Proof. (1) To show $\left\{u_{n}+h\left(\sigma_{n} u_{n}+\pi\right)\right\} \in \partial M_{R}$ it is sufficient to prove $\int_{G} F\left(u_{n}+h\left(\sigma_{n} u_{n}+\pi\right), x\right)=R$. As $\left\{u_{n}\right\}$ are elements of $\partial M_{R}$, we show 


$$
\int_{G}\left[F\left(u_{n}+h\left(\sigma_{n} u_{n}+\pi\right), x\right)-F\left(u_{n}, x\right)\right] \mathrm{I}=0 .
$$

Using Lemma III.1.2 and Fubini's theorem, we obtain from (1)

$$
\int_{0}^{1} d s \int_{G}\left(\sigma_{n} u_{n}+\pi_{n}\right) f\left[u_{n}+\operatorname{sh}\left(\sigma_{n} u_{n}+\pi\right)\right]=0 .
$$

Denote the expression on the left by $\mathscr{G}\left(\sigma_{n}\right)$. We shall show that for $|h|<h_{\varepsilon}$, with $h_{\varepsilon}$ sufficiently small and independent of $n$, there is a real number $\sigma_{n}$ such that $\mathscr{G}\left(\sigma_{n}\right)=0$ and hence the first conclusion of the lemma will follow.

First, by Lemma II.3.3,

$$
\mathscr{G}(\sigma)=\int_{0}^{1}\left\langle\sigma u_{n}+\pi_{1}, B\left(u_{n}+\operatorname{sh}\left(\sigma u_{n}+\pi\right)\right)\right\rangle_{m, 2} d s .
$$

As the operator $B$ is continuous, $\mathscr{G}(\sigma)$ is a continuous real-valued function of $\sigma$. Next rewriting $\mathscr{G}(\sigma)$,

$$
\begin{aligned}
\mathscr{G}(\sigma)= & \left\langle u_{n}, B\left(u_{n}\right)\right\rangle_{m, 2}+\left\langle\pi, B u_{n}\right\rangle_{m, 2} \\
& +\int_{0}^{1}\left\langle\sigma u_{n}+\pi, B\left(u_{n}+\operatorname{sh}\left(\sigma u_{n}+\pi\right)\right)-B\left(u_{n}\right)\right\rangle_{m, 2} d s .
\end{aligned}
$$

The last term of the right-hand side will be denoted by $H(\sigma)$. We show now that $H(\sigma)$ can be made arbitrarily small for sufficiently small $h$, if $\sigma$ is restricted to the interval $[-M, M]$, where $M$ is a number to be determined independent of $n$. In this case, the set of elements $\left\{u_{n}+\operatorname{sh}\left(\sigma u_{n}+\pi\right)\right\}$ is uniformly bounded in $\mathscr{W}_{2}^{(m)}(G)$ and, by Sobolev's Imbedding Theorem is a precompact set in $L_{p}(G)$ for $1 \leqq p<2 N /(N-2 m)$ if $N>2 m ; 1 \leqq p<\infty$ otherwise. We consider the case $N>2 m$ (the case $N \leqq 2 m$ is completely analogous). By the polynomial growth condition on $f(t, x)$ and Vainberg's Theorem I.6.1, the mapping $\bar{f}(u)=f[u(x), x]$ from $L_{p} \rightarrow L_{2 N /(N+2 m)}$ is continuous $(1 \leqq p<2 N /(N-2 m))$. Thus by restricting $\bar{f}$ to the closure of the precompact set $\left\{u_{n}+\operatorname{sh}\left(\sigma u_{n}+\pi_{n}\right)\right\}$ in $L_{p}$, we can say $f$ is uniformly continuous.

By Hölder's inequality,

$$
|H(\sigma)|
$$

$$
\leqq\left\|\sigma u_{n}+\pi\right\|_{L_{2 N /(N-2 m)}} \int_{0}^{1}\left\|f\left(u_{n}+\operatorname{sh}\left(\sigma u_{n}+\pi\right), x\right)-f\left(u_{n}, x\right)\right\|_{L_{2 N /(N+2 m)}} d s .
$$

Using Sobolev's Imbedding Theorem, the set $\left\{\sigma u_{n}+\pi\right\}$ is uniformly bounded in $L_{2 N /(N-2 m)}$. Hence by (2) and the uniform continuity of $f$, given $\varepsilon>0$, we can determine a number $h_{\varepsilon}>0$ such that whenever $\left\|s h\left(\sigma u_{n}+\pi\right)\right\|_{L_{p}}<h_{\varepsilon},|H(\sigma)|<\varepsilon$. Now as the $L_{p}$ norms of the set $\left\{\sigma u_{n}+\pi\right\}$ are uniformly bounded by $M_{2}$, say, and $0 \leqq s \leqq 1$, if we set $M_{2}|h|<h_{\varepsilon},|H(\sigma)|<\varepsilon$ for any $\sigma \in[-M, M]$. Set $h_{\varepsilon}=M_{2} h_{\varepsilon}$. Now 


$$
\mathscr{G}(\sigma)=\sigma\left\langle u_{n}, B u_{n}\right\rangle_{m, 2}+\left\langle\pi, B u_{n}\right\rangle_{m, 2}+H(\sigma) .
$$

Suppose $\varepsilon>0$ is given and $|h|$ is chosen so small that $|H(\sigma)|<\varepsilon$. Let

$$
\sigma_{N}^{\prime}=\frac{1}{\left\langle u_{n}, B u_{n}\right\rangle_{m, 2}}\left(-\left\langle\pi, B u_{n}\right\rangle_{m, 2}+\varepsilon\right)
$$

then from (3) $\mathscr{G}\left(\sigma_{n}^{\prime}\right)=\varepsilon+H\left(\sigma_{n}^{\prime}\right)$. Hence, if $\sigma_{n}^{\prime} \in[-M, M],\left|H\left(\sigma_{n}^{\prime}\right)\right|<\varepsilon$ and $\mathscr{G}\left(\sigma_{n}^{\prime}\right)>0$. Let

$$
\sigma_{n}^{\prime \prime}=\frac{1}{\left\langle u_{n}, B u_{n}\right\rangle_{m, 2}}\left(-\left\langle\pi, B u_{n}\right\rangle_{m, 2}-\varepsilon\right) ;
$$

then, from (3), $\mathscr{G}\left(\sigma_{n}^{\prime \prime}\right)=-\varepsilon+H\left(\sigma_{n}^{\prime \prime}\right)$. If $\sigma_{n}^{\prime \prime} \in[-M, M],\left|H\left(\sigma_{n}^{\prime \prime}\right)\right|<\varepsilon$ and hence $\mathscr{G}\left(\sigma_{n}^{\prime \prime}\right)<0$. As $\mathscr{G}(\sigma)$ is a continuous real-valued function of $\sigma$ we conclude that under the given conditions for some $\sigma_{n}$ between $\sigma_{n}^{\prime}$ and $\sigma_{n}^{\prime \prime}, \mathscr{G}\left(\sigma_{n}\right)=0$.

To complete the first part of the lemma we show that a number $M$ can be chosen, independent of $n$, such that the set of real numbers $\left\{\sigma_{n}^{\prime}, \sigma_{n}^{\prime \prime}\right\}$ lies in $[-M, M]$. First we note that as $\left\{u_{n}\right\} \in \partial M_{R}$,

$$
\left\langle u_{n}, B u_{n}\right\rangle=\int_{G} u_{n} f\left(u_{n}, x\right) \geqq K \int_{G} F\left(u_{n}, x\right)=K R .
$$

Next as $B$ is a compact mapping the set $\left\{B u_{n}\right\}$ is uniformly bounded in $\mathscr{W}_{2}^{(m)}(G)$ by $M_{3}$, say. It suffices to consider $\left\{\sigma_{n}^{\prime \prime}\right\}$. Using Schwarz's inequality and (5)

$$
\begin{aligned}
\left|\sigma_{n}^{\prime \prime}\right| & \leqq\left\langle u_{n}, B u_{n}\right\rangle_{m, 2}^{-1}\left(\|\pi\|_{m, 2}\left\|B u_{n}\right\|_{m, 2}+\varepsilon\right) \\
& \leqq(K R)^{-1}\left(M_{3}\|\pi\|_{m, 2}+\varepsilon\right) \\
& \leqq M \quad \text { (independent of } n) .
\end{aligned}
$$

(2) The second part of the lemma gives an estimate for the numbers $\sigma_{n}$, independent of $h$. In the first part of the lemma we have shown that $\sigma_{n}$ lies between $\sigma_{n}^{\prime}$ and $\sigma_{n}^{\prime \prime}$. Recalling the values of $\sigma_{n}^{\prime}$ and $\sigma_{n}^{\prime \prime}$ from (4) and (5) we obtain

$$
\begin{aligned}
\left|\sigma_{n}+\frac{\left\langle\pi, B u_{n}\right\rangle_{m, 2}}{\left\langle u_{n}, B u_{n}\right\rangle_{m, 2}}\right| & \leqq \frac{2 \varepsilon}{\left\langle u_{n}, B u_{n}\right\rangle_{m, 2}} \\
& \leqq\left(\frac{2}{K R}\right) \varepsilon<K \varepsilon
\end{aligned}
$$

where $R$ is a positive constant independent of $n$ and $\varepsilon$.

IV. Existence theory-variational method. The object of this section is to prove the following theorem.

THEOREM IV.1 (EXISTENCE THEOREM). Let $G$ be any bounded domain in $R^{N}$. Then the boundary value problem (II.1.1) has a generalized eigenfunction $u(x)$, if $f(t, x)$ is in the class $\mathscr{F}(\rho)$, where 


$$
\begin{array}{ll}
0 \leqq \rho<\frac{N+2 m}{N-2 m}, & N>2 m, \\
0 \leqq \rho<\infty, & N \leqq 2 m .
\end{array}
$$

The generalized eigenfunction $u(x)$ is normalized by the requirement that $u(x) \in \partial M_{R}$ for fixed positive $R$ and characterized as a solution of the variational problem inf $\langle\mathfrak{A} v ; v\rangle_{m, 2}$ for $v \in \partial M_{R}$.

The proof of this result will be carried out in a sequence of seven lemmas. Throughout the discussion we shall assume $R$ is a fixed positive number and $\partial M_{R}$ is constructed as in $§ I I I$.

IV.1. Proof of the existence theorem.

LEMMA IV.1.1. For $\left.u \in \partial M_{R},\langle\mathfrak{A} u, u\rangle_{m, 2} \geqq k_{R}\right\rangle-\infty$ where $k_{R}$ is a constant independent of $u$.

Proof. By Lemma II.3.2 (Gårding's inequality) for all functions $u \in \mathscr{W}_{2}^{(m)}(G)$

$$
\langle\mathfrak{A} u, u\rangle_{m, 2} \geqq k_{1}\|u\|_{m, 2}^{2}-k_{2}\|u\|_{0,2}^{2} \text {. }
$$

If $k_{2}=0$, the lemma is, of course, immediate. Otherwise, by Lemma I.4.3 for any $\varepsilon>0$ and $u \in \mathscr{W}_{2}^{(m)}(G)$

$$
\|u\|_{0,2}^{2} \leqq 2 \varepsilon\|u\|_{m, 2}^{2}+2 K(\varepsilon)\|u\|_{0,1}^{2}
$$

Eliminating $\|u\|_{0,2}^{2}$ from (1) and (2) we obtain

$$
\langle\mathfrak{U} u, u\rangle_{m, 2} \geqq\left(k_{1}-2 \varepsilon k_{2}\right)\|u\|_{m, 2}^{2}-2 K_{0} K(\varepsilon)\|u\|_{1,1}^{2} .
$$

Choosing $k_{1}=2 \varepsilon_{1} k_{2},\langle\mathfrak{A} u, u\rangle_{m, 2} \geqq-2 K_{0} K\left(\varepsilon_{1}\right)\|u\|_{0,1}^{2}$. On the other hand, by Lemma III.3.1, the $L_{1}$-boundedness lemma, for functions of $\partial M_{R},\|u\|_{0,1} \leqq g(R)$. Thus $\langle\mathfrak{A} u, u\rangle_{m, 2} \geqq-2 K_{0} K\left(\varepsilon_{1}\right) g^{2}(R)=k_{r}>-\infty$.

LEMMA IV.1.2. Any minimizing sequence for the variational problem $\inf _{u \in \partial M_{R}}\langle\mathfrak{A} u, u\rangle_{m, 2}$ has uniformly bounded $W_{2}^{m}(G)$ norms.

Proof. By Lemma IV.1.1, $\langle\mathfrak{A} u, u\rangle_{m, 2} \geqq k_{R}$, for $u \in \partial M_{R}$, i.e. the set of numbers $\langle\mathfrak{U} u, u\rangle_{m, 2}$ is bounded below for $u \in \partial M_{R}$. Thus $\inf _{\partial M_{R}}\langle\mathfrak{U} u, u\rangle_{m, 2}$ is a well-defined number which we denote by $l(R)$. Let $\left\{u_{n}\right\}$ be a minimizing sequence for this variational problem chosen from the set $\partial M_{R}$, i.e. $\lim _{n \rightarrow \infty}\left\langle\mathfrak{A} u_{n}, u_{n}\right\rangle_{m, 2}=l(R)$. Hence for sufficiently large $n$,

$$
[l(R)+1] \geqq\left\langle\mathfrak{A} u_{n}, u_{n}\right\rangle_{m, 2} .
$$

Using Lemma II.3.2 (Gårding's inequality),

$$
\left\langle\mathcal{U} u_{n}, u_{n}\right\rangle_{m, 2} \geqq k_{1}\left\|u_{n}\right\|_{m, 2}^{2}-k_{2}\left\|u_{n}\right\|_{0,2}^{2} .
$$

If $k_{2}=0$, (1) and (2) clearly imply the lemma; otherwise, 


$$
K_{2}\left\|u_{n}\right\|_{0,2}^{2}+[l(R)+1] \geqq k_{1}\left\|u_{n}\right\|_{m, 2}^{2} .
$$

Using Lemma 1.4.3, and the $L_{1}$-boundedness lemma, with $\varepsilon=K_{2} /\left(1+K_{1}\right)$, as in the previous lemma, we obtain, for sufficiently large $n$, that $\left\|u_{n}\right\|_{W_{2}^{m}(G)} \leqq g_{2}(R)$, where $g_{2}(R)$ is a constant independent of $n$.

LEMMA IV.1.3. Let $\left\{u_{n}\right\}$ be a minimizing sequence for the variational problem $\inf _{M_{R}}\langle\mathfrak{A} u, u\rangle_{m, 2}$. Then $\left\{u_{n}\right\}$ has a subsequence, which we again label $\left\{u_{n}\right\}$, with the following properties:

(1) $u_{n} \rightarrow u$ weakly in $\mathscr{W}_{2}^{(m)}(G)$.

(2) $u_{n} \rightarrow u$ strongly in $L_{2}(G)$.

(3) $u_{n} \rightarrow u$ a.e. in $G$.

(4) $\left.\left\langle B u_{n}, u_{n}\right\rangle_{m, 2} \rightarrow\langle B u, u\rangle_{m, 2}\right\rangle 0$.

(5) $u \in \partial M_{R}$ and hence $u \neq 0$.

Proof. (1) By Lemma IV.1.2 for sufficiently large $n,\left\|u_{n}\right\|_{m, 2} \leqq g_{2}(R)$ and as $\mathscr{W}_{2}^{(m)}(G)$ is a Hilbert space, $\left\{u_{n}\right\}$ has a weakly convergent subsequence, which we again label $\left\{u_{n}\right\}$, such that $u_{n} \rightarrow u$ weakly in $\mathscr{W}_{2}^{(m)}(G)$ where $u$ is a uniquely determined element of $\mathscr{W}_{2}^{(m)}(G)$.

(2) This result is an immediate consequence of the corollary to Sobolev's Imbedding Theorem, and (1).

(3) As $u_{n} \rightarrow u$ strongly in $L_{2}(G),\left\{u_{n}\right\}$ can be refined to a subsequence, which we again label $\left\{u_{n}\right\}$, such that $u_{n} \rightarrow u$ a.e. in $G$.

(4) This result is an immediate consequence of (1) and the fact that $\langle B u, v\rangle_{m, 2}$ is a weakly continuous function of $u, v \in \mathscr{W}_{2}^{(m)}(G)$.

(5) As $u_{n} \in \partial M_{R}$, and $u_{n} \rightarrow u$ weakly, by Lemma III.3.3, $u \in \partial M_{R}$. By Lemma III.2.1 (4), $u \neq 0$ in $\mathscr{W}_{2}^{(m)}(G)$. Thus $\left.\langle B u, u\rangle_{m, 2}\right\rangle 0$.

LEMMA IV.1.4. Let $\left\{u_{n}\right\}$ be the sequence referred to in Lemma IV.1.3. Let $\pi$ be an arbitrary element of $\mathscr{W}_{2}^{(m)}(G)$. Then $\lim _{n \rightarrow \infty}\left\langle\mathfrak{A} u_{n}, b_{n} u_{n}+\pi\right\rangle_{m, 2}=0$, where $-b_{n}=\left\langle B u_{n}, \pi\right\rangle_{m, 2} /\left\langle B u_{n}, u_{n}\right\rangle_{m, 2}$.

Proof. For some fixed $\varepsilon>0$, by the Variational Lemma III.3.4 for each $u_{n}$ of the refined minimizing sequence of Lemma IV.1.3, we obtain a new sequence $\left\{u_{n}+h\left(\sigma_{n} u_{n}+\pi\right)\right\} \in \partial M_{R}$, for each $h,|h|<h_{\varepsilon}$. Also $\left|\sigma_{n}-b_{n}\right|<R \varepsilon$ where $R$ is independent of $n$ and $\varepsilon$.

$$
\text { As inf }_{\partial M_{R}}\langle\mathfrak{U} u, u\rangle_{m, 2} \geqq l(R) \text {, }
$$

$$
\left\langle\mathfrak{U}\left(u_{n}+h\left(\sigma_{n} u_{n}+\pi\right)\right), u_{n}+h\left(\sigma_{n} u_{n}+\pi\right)\right\rangle_{m, 2} \geqq l(R) .
$$

Hence, as $\mathfrak{A}$ is self-adjoint,

$$
\begin{aligned}
\left(\left\langle\mathfrak{A} u_{n}, u_{n}\right\rangle_{m, 2}-l(R)\right) & +2 h\left\langle\mathfrak{U} u_{n}, \sigma_{n} u_{n}+\pi\right\rangle_{m, 2} \\
& +h^{2}\left\langle\mathfrak{U}\left(\sigma_{n} u_{n}+\pi\right), \sigma_{n} u_{n}+\pi\right\rangle_{m, 2} \geqq 0 .
\end{aligned}
$$


We now consider the left-hand side of this inequality. As $\left\{u_{n}\right\}$ is a minimizing sequence, the first term tends to 0 , and the third term can be dominated by $h^{2} M$, where $M$ is a positive constant independent of $n$ and $h$. Thus we can write from (1)

$$
2 h\left\langle\mathfrak{A} u_{n}, \sigma_{n} u_{n}+\pi\right\rangle_{m, 2}+h^{2} M_{1} \geqq 0 .
$$

Now as $n \rightarrow \infty$, we consider

$$
\left\langle\mathfrak{U} u_{n}, \sigma_{n} u_{n}+\pi\right\rangle_{m, 2}=\left\langle\mathfrak{U} u_{n}, b_{n} u_{n}+\pi\right\rangle_{m, 2}+\left(\sigma_{n}-b_{n}\right)\left\langle\mathfrak{U} u_{n}, u_{n}\right\rangle_{m, 2} .
$$

As $n \rightarrow \infty,\left\langle\mathfrak{A} u_{n}, b_{n} u_{n}+\pi\right\rangle_{m, 2} \rightarrow s$, where $s$ is constant independent of $h$; also for each $h,|h|<h_{\varepsilon}$, we have $\left|\left(\sigma_{n}-b_{n}\right)\left\langle\mathfrak{A} u_{n}, u_{n}\right\rangle_{m, 2}\right| \leqq R(l(R)+1) \varepsilon$.

Suppose $s \neq 0$. Choose $\varepsilon$ so small that $\left\langle\mathfrak{U} u_{n}, \sigma_{n} u_{n}+\pi\right\rangle_{m, 2}=s+r_{n}$ where $\left|r_{n}\right|<|s| / 2$. Thus from (2)

$$
\frac{s+r_{n}}{h}+M_{1} \geqq 0
$$

Hence as $h$ varies over $\left(-h_{\varepsilon}, h_{\varepsilon}\right)$, the inequality (3) will be violated. Therefore we conclude $s=0$.

LEMmA IV.1.5. Let $u$ be the weak limit of the sequence $\left\{u_{n}\right\}$ of IV.1.4. Then $u$ is an eigenfunction of the equation $\mathfrak{A} u-\lambda B u=0$ where $\lambda=l(R) /\langle B u, u\rangle_{m, 2}$.

Proof. Let $\pi$ be an arbitrary element of $W_{2}^{(m)}(G)$. Thus, by Lemma IV.1.4, $\left\langle\mathfrak{A} u_{n}, \pi\right\rangle+b_{n}\left\langle\mathfrak{A} u_{n}, u_{n}\right\rangle=0$. Letting $n \rightarrow \infty$ and noting that $a_{n} \rightarrow u$ weakly we obtain $\langle\mathfrak{U} u, \pi\rangle_{m, 2}-\left(l(R) /\langle B u, u\rangle_{m, 2}\right)\langle B u, \pi\rangle_{m, 2}=0$, i.e.

$$
\langle\mathfrak{A} u-\lambda B u, \pi\rangle_{m, 2}=0, \text { where } \frac{l(R)}{\langle B u, u\rangle_{m, 2}} .
$$

As the first term of the inner product in (1) is independent of $\pi$, (1) holds for all $\pi \in \mathscr{W}_{2}^{(m)}(G)$. As $\mathscr{W}_{2}^{(m)}(G)$ is a Hilbert space, $\mathfrak{A} u-\lambda B u=0$ and as $u \neq 0$ in $\mathscr{W}_{2}^{(m)}(G), u$ is an eigenfunction for the operator equation $\mathfrak{A} u-\lambda B u=0$. Also, by Lemma IV.1.3, $u \in \partial M_{R}$.

LEMMA IV.1.6. Let $\left\{u_{n}\right\}$ be the refined minimizing sequence referred to in Lemma IV.1.4. Then $u_{n} \rightarrow u$ strongly in $\mathscr{W}_{2}^{(m)}(G)$.

Proof. The results of Lemmas IV.1.3-5 show the following four limits hold:

$$
\begin{array}{r}
\lim _{n \rightarrow \infty}\left(\left\langle\mathfrak{A} u_{n}, u\right\rangle_{m, 2}-\lambda\left\langle B u_{n}, u\right\rangle_{m, 2}\right)=0 . \\
\lim _{n \rightarrow \infty}\left(\langle\mathfrak{U} u, u\rangle_{m, 2}-\lambda\langle B u, u\rangle_{m, 2}\right)=0 . \\
\lim _{n \rightarrow \infty}\left(\left\langle\mathfrak{A} u_{n}, u_{n}\right\rangle_{m, 2}-\lambda\left\langle B u_{n}, u_{n}\right\rangle_{m, 2}\right)=0 . \\
\lim _{n \rightarrow \infty}\left(\left\langle\mathfrak{A} u, u_{n}\right\rangle-\lambda\left\langle B u, u_{n}\right\rangle_{m, 2}\right)=0 .
\end{array}
$$


From (1)-(2), we obtain

$$
\lim _{n \rightarrow \infty}\left\langle\mathfrak{A}\left(u_{n}-u\right), u\right\rangle_{m, 2}=0 .
$$

From (3)-(4), we obtain

$$
\lim _{n \rightarrow \infty}\left\langle\mathfrak{U}\left(u_{n}-u\right), u_{n}\right\rangle_{m, 2}=0
$$

From (5)-(6), we obtain

$$
\lim _{n \rightarrow \infty}\left\langle\mathfrak{A}\left(u_{n}-u\right), u_{n}-u\right\rangle_{m, 2}=0 .
$$

From Lemma II.3.2 (Gårding's inequality)

$$
\left\langle\mathfrak{A}\left(u_{n}-u\right), u_{n}-u\right\rangle_{m, 2}+k_{2}\left\|u_{n}-u\right\|_{0,2}^{2} \geqq k_{1}\left\|u_{n}-u\right\|_{m, 2}^{2} .
$$

By Lemma IV.1.3, $u_{n} \rightarrow u$ strongly in $L_{2}$; thus by (7) $\left\|u_{n}-u\right\|_{m, 2} \rightarrow 0$, i.e., $u_{n} \rightarrow u$ strongly in $\mathscr{W}_{2}^{(m)}(G)$.

LEMMA IV.1.7. Let $u$ be the eigenfunction of the operator equation $\mathfrak{U} u-\lambda B u=0$ obtained in Lemma IV.1.5. Then $u$ is a solution of the variational problem $\inf _{\partial M_{R}}\langle\mathfrak{A} v, v\rangle_{m, 2} ; u$ is also a generalized eigenfunction of the boundary value problem (II.1.1) as stated in Theorem IV.1.

Proof. Let $\left\{u_{n}\right\}$ be the minimizing sequence for the variational problem $\inf _{\partial M_{R}}\langle\mathfrak{A} v, v\rangle_{m, 2}$ referred to in Lemma IV.1.6. Hence $u_{n} \rightarrow u$ strongly.

$\left\langle\mathfrak{U} u_{n}, u_{n}\right\rangle_{m, 2} \rightarrow\langle\mathfrak{A} u, u\rangle_{m, 2}=l(R)$. Thus $u$ is a solution of the variational problem $\inf _{\partial M_{R}}\langle\mathfrak{U} u, u\rangle_{m, 2}$. The fact that $u$ is a generalized eigenfunction for (II.1.1) follows from Theorem II.3.1 and Lemma IV.1.5.

Corollary to THEOREM IV.1. Suppose $f(u, x)=g(x) u^{2 k+1}$, for some integer $k$, is in the class $\mathscr{F}(\rho)$ of Theorem IV.1. Then the associated eigenvalue, $\lambda$, of the eigenfunction characterized by Theorem IV.1 is the first eigenvalue on $\partial M_{R}$ (i.e., for any other eigenvalue $\lambda^{\prime}$, whose associated eigenfunction $u^{\prime} \in \partial M_{R}$, $\left.\lambda \leqq \lambda^{\prime}\right)$.

Proof. Suppose $\lambda$ and $\lambda^{\prime}$ are two eigenvalues with associated eigenfunctions $u$ and $u^{\prime} \in \partial M_{R}$ for the operator equation $\mathfrak{A} u-\lambda B u=0$; then

$$
\begin{aligned}
\lambda\langle u, B u\rangle_{m, 2} & =\inf _{\partial M_{R}}\langle\mathfrak{A} u, u\rangle=l(R), \\
\lambda^{\prime}\left\langle u^{\prime}, B u^{\prime}\right\rangle_{m, 2} & =\left\langle\mathfrak{A} u^{\prime}, u^{\prime}\right\rangle \geqq l(R) .
\end{aligned}
$$

Thus $\lambda^{\prime}\left\langle u^{\prime}, B u^{\prime}\right\rangle_{m, 2} \geqq \lambda\langle u, B u\rangle_{m, 2}$. As $u, u^{\prime} \in \partial M_{R},\left\langle u^{\prime}, B u^{\prime}\right\rangle_{m, 2}=\langle u, B u\rangle_{m, 2}$. Thus $\lambda^{\prime} \geqq \lambda$.

V. Regularity theory. In this section we study the smoothness restrictions that must be placed on the boundary value problem (II.1.1) to guarantee that the 
generalized eigenfunction obtain€d in $§ I V$ is, in fact, a classical solution. Indeed, given the boundary value problem II.1.1 :

$$
\begin{aligned}
A u-\lambda f(u, x) & =0, \\
\left.u\right|_{\partial G} & =\left.D u\right|_{\partial G}=\cdots=\left.D^{m-1} u\right|_{\partial G}=0,
\end{aligned}
$$

we shall assume the regularity conditions:

I(a) $G$ is of class $4 m$.

I(b) For the coefficients of $A, a_{\alpha \beta}(x) \in C^{2 m}(\bar{G})$.

I(c) $f(t, x)$ satisfies a local Lipschitz condition in $t$ for $t \in R^{\prime}$, and a local Hölder condition of exponent $\gamma, 0<\gamma<1$, for $x \in G$.

We shall divide the regularity theory into three parts:

(1) Regularity in the interior of the bounded domain $G$. It is shown that under the regularity conditions above, any generalized solution $u(x)$ of (II.1.1) is in $C^{2 m}(G)$.

(2) Regularity at the boundary of the bounded domain $G$. It is shown that $u(x) \in C^{m-1}(\bar{G})$.

(3) Assumption of boundary values. It is shown that $\left.u\right|_{\partial G}=\left.D u\right|_{\partial G}=\cdots$ $=\left.D^{m-1} u\right|_{\partial G}=0$, in the pointwise sense.

Throughout this section we assume $f(t, x) \in \mathscr{F}(\rho)$ with

$$
\begin{array}{ll}
0 \leqq \rho<\frac{N+2 m}{N-2 m}, & N>2 m, \\
0 \leqq \rho<\infty, & N \leqq 2 m .
\end{array}
$$

We summarize these results as follows:

THEOREM V.1. Let $u(x)$ be any generalized solution of the boundary value problem (II.1.1) where $f(t, x)$ is in the class $\mathscr{F}(\rho)$ for

$$
\begin{array}{ll}
0 \leqq \rho<\frac{N+2 m}{N-2 m}, & N>2 m, \\
0 \leqq \rho<\infty, & N \leqq 2 m .
\end{array}
$$

Suppose the regularity conditions $\mathrm{I}(\mathrm{a}), \mathrm{I}(\mathrm{b})$ and $\mathrm{I}(\mathrm{c})$ hold. Then $u(x)$ is, indeed, a classical solution of (II.1.1).

V.1. Transition from $\mathscr{W}_{2}^{(m)}(G)$ to $W_{p}^{2 m}(G)$. The proof of Theorem V.1 will be based on the $L_{p}$ regularity theory for linear nonhomogeneous elliptic equations $A u=f$, developed in recent years by Browder [5], [6], Koshelev [19], Agmon [1], and Agmon-Douglis-Nirenberg [2]. In particular the following result is of prime importance for the nonlinear theory:

THEOREM V.1.1. Let $G$ be a bounded domain of class $C^{2 m}$ in $R^{N}$. Suppose the elliptic operator $A$ satisfies the regularity condition $\mathrm{I}(\mathrm{b})$ and has the properties 
described in II.1(d). Suppose $u(x)$ is a generalized solution, in $L_{p}(G)(1<p<\infty)$, of the following boundary value problem:

$$
\begin{aligned}
& A u=f(x) \text { in } G, \\
& D^{\alpha} u=0 \text { on } \partial G, \quad 0 \leqq|\alpha| \leqq m-1 .
\end{aligned}
$$

If $f(x) \in L_{p}(G)(1<p<\infty)$, then $u(x) \in W_{p}^{2 m}(G)$ with $\|u\|_{2 m, p} \leqq c\left\{\|f\|_{0, p}+\|u\|_{0, p}\right\}$, where $c$ is a positive constant independent of $u$.

For the proof of this result we refer to the paper of Agmon [1, Theorem 8.2]. Applying Theorem V.1.1 to the present situation, we obtain:

LEMMA V.1.2. Let $u(x)$ be any generalized solution of the boundary value problem (II.1.1). Suppose the regularity conditions $\mathrm{I}(\mathrm{a}), \mathrm{I}(\mathrm{b})$ are satisfied. Then $u(x) \in W_{p}^{2 m}(G)$ with

$$
p=\left\{\begin{array}{l}
\frac{2 N}{N+2 m}(1+\varepsilon) \text { for some } \varepsilon>0 \text { if } N>2 m, \\
2 \quad \text { if } N \leqq 2 m .
\end{array}\right.
$$

Proof. As $u(x)$ is a generalized solution of the boundary value problem (II.1.1), $u(x) \in \mathscr{W}_{2}^{(m)}(G)$. Set $\tilde{f}(x)=f(u(x), x)$.

Case I $(N \leqq 2 m)$. By Sobolev's Imbedding Theorem and the polynomial growth condition on $f(t, x), \tilde{f}[x] \in L_{2}(G)$; hence setting $p=2$, by Theorem V.1.1, $u \in W_{2}^{2 m}(G)$.

Case II (a) $(N>2 m) . f(t, x) \in \mathscr{F}(\rho), 0 \leqq \rho \leqq N /(N-2 m)$. Here, again, by the polynomial growth condition on $f(t, x)$ and Sobolev's Imbedding Theorem $\bar{f}[x] \in L_{2}(G)$ and, by Theorem V.1.1 with $p=2, u \in W_{2}^{2 m}(G)$.

Case II (b) $(N>2 m) . f(t, x) \in \mathscr{F}(\rho), N /(N-2 m)<\rho<2 N /(N-2 m)$. Here, set $(1+\varepsilon) \rho=(N+2 m) /(N-2 m)$, when $\varepsilon$ is a fixed number, $0<\varepsilon<2 m / N<1$. Thus, by Sobolev's Imbedding Theorem and the polynomial growth condition on $f(t, x), \tilde{f}(x) \in L_{2 N \cdot(1+\varepsilon) /(N+2 m)}$. Hence, by Theorem V.1.1, $u \in W_{p}^{2 m}(G)$, $p=2 N \cdot(1+\varepsilon) /(N+2 m)$.

V.2. Interior regularity. The following two results of Browder [6, Theorems $2.11,2.12]$ are basic for our procedure.

THEOREM V.2.1. Let $A$ be a linear differential operator of order $2 m$ defined on $G$ with top order coefficients in $C_{0}(G)$ and all of its coefficients essentially bounded on each compact subset of $G$. Suppose $1<p<p_{1}<\infty$, and $u \in W_{p}^{2 m}\left(G^{\prime}\right)$ and $A u \in L_{p_{1}}\left(G^{\prime}\right)$; then $u \in W_{p_{1}}^{2 m}\left(G^{\prime}\right)$ for any subdomain $G^{\prime}$ with compact closure in $G$.

THEOREM V.2.2. Let $A$ be a linear elliptic differential operator of order $2 m$ defined on $G$ with coefficients in $C^{0, \gamma}\left(G^{\prime}\right)$ for any subdomain $G^{\prime}$ with compact closure in $G$. Let $u \in W_{p}^{2 m}\left(G^{\prime}\right)(1<p<\infty)$ and $A u \in C^{0, \gamma}\left(G^{\prime}\right)(0<\gamma<1)$. Then $u$ lies in $C^{2 m, \gamma}\left(G^{\prime}\right)$. 
Applying these results to our context, we obtain:

LEMMA V.2.3. Let $u(x)$ be any generalized solution of the boundary value problem (II.1.1). Suppose the regularity conditions I(b), I(c) are satisfied. Then $u(x) \in C^{2 m}(G)$.

Proof. First we shall prove $u(x) \in W_{p}^{2 m}\left(G^{\prime}\right)$ for any subdomain $G^{\prime}$ of $G$, with compact closure in $G$. In case $N \leqq 2 m$, this result is quickly obtained. For, by Lemma V.1.2, $u(x) \in W_{2}^{2 m}(G)$; thus $\bar{f}(x)=f(u(x), x)$ lies in $L_{p}(G)$ for any $p<\infty$ by Sobolev's Imbedding Theorem. Hence, by Theorem V.2.1, $u(x) \in W_{p}^{2 m}\left(G^{\prime}\right)$ for any $G^{\prime}$ and $p(1<p<\infty)$.

In case $N>2 m$, we carry out a so-called bootstrap procedure. By Lemma V.1.2, $u(x) \in W_{p}^{2 m}(G)$ for some $p=2 N \cdot(1+\varepsilon) /(N+2 m)$. By Sobolev's Imbedding Theorem $\bar{f}[x]=f(u(x), x) \in L_{p_{1}}(G)$ where $1 / p_{1}=1 / p-2 m / N$. Thus by Theorem V.2.1, $u(x) \in W_{p_{1}}^{2 m}\left(G^{\prime}\right)$ as $p_{1}>p$. In general, by carrying out this procedure $s$ times we obtain a sequence of numbers $\left\{p_{s}\right\}$ tending monotonely to $\infty$ with $u(x) \in W_{p_{s}}^{2 m}\left(G^{\prime}\right)$. If at any stage $N<2 m p_{s}$, by Sobolev's Imbedding Theorem $\tilde{f}(x) \in L_{p}\left(G^{\prime}\right)$ for any $p, 1<p<\infty$, and, by Theorem V.2.1, $u \in W_{p}^{2 m}\left(G^{\prime}\right)$ for any $p, 1<p<\infty$.

Next we show $u(x) \in C^{0, \gamma}\left(G^{\prime}\right)$ for some $\gamma, 0<\gamma<1$. This result follows immediately from the third part of the Sobolev Imbedding Theorem as

$$
W_{p}^{2 m}\left(G^{\prime}\right) \rightarrow C^{0, \gamma}\left(G^{\prime}\right),
$$

for sufficiently large $p$, is a continuous imbedding.

Thus $\bar{f}[x]=f(u(x), x)$ satisfies a Hölder condition in $G^{\prime}$ of exponent $\gamma_{1} 0<\gamma_{1}<1$, by virtue of the regularity condition $\mathrm{I}(\mathrm{c})$ of $f(t, x)$. Thus, by Theorem V.2.2, $u \in C^{2 m, \gamma_{1}}\left(G^{\prime}\right)$ and as $G^{\prime}$ is an arbitrary subdomain of $G, u \in C^{2 m}(G)$.

V.3. Regularity at the boundary. The following result, modified from Browder [5, Theorem 14], is basic for our procedure.

TheOREM V.3.1. Let $G$ be a bounded domain of class $C^{4 m}$, and suppose $A$ is a real formally self-adjoint elliptic operator of order $2 m$, satisfying regularity condition I(b). Then if $u \in W_{p}^{(m)}(G) \cap W_{p}^{2 m}(G)$ and $A u \in L_{p_{1}}(G)$ with $1<p \leqq p_{1}<\infty, u \in W_{p 1}^{(m)}(G) \cap \mathscr{W}_{p_{1}}^{2 m}(G)$.

Applying this result to our context we obtain

LEMMA V.3.2. Let $u(x)$ be any generalized solution of the boundary value problem (II.1.1). Suppose the regularity conditions I(a), (b), (c) are satisfied. Then $u(x) \in C^{m-1}(\bar{G})$.

Proof. First we prove $u(x) \in W_{p}^{2 m}(G)$ for any $1 \leqq p<\infty$. If we use Theorem V.3.1 in place of Theorem V.2.1, we can follow the method used in Lemma V.2.3 in a step-by-step manner. Thus by Sobolev's Imbedding Theorem ( $\S I$ I) there is 
a continuous imbedding $W_{p}^{2 m}(G) \rightarrow C^{m-1}(\bar{G})$, for sufficiently large $p$. Thus $u(x) \in C^{m-1}(\bar{G})$.

V.4. Assumption of boundary values.

Lemma V.4.1. Suppose $u(x) \in \mathscr{W}_{2}^{(1)}(G) \cap C(\vec{G})$ and $G$ is of class $C^{\prime}$; then $u(x) \equiv 0$ on $\partial G$ in the pointwise sense.

For the proof of this lemma, we refer to the paper of Nirenberg [29].

An immediate consequence of Lemma V.4.1 is the result mentioned in Lemma II.2.1.

LEMMA V.4.2. Suppose $u(x)$ is any generalized solution of the boundary value problem (II.1.1). Suppose $u(x) \in C^{2 m}(G) \cap C^{m-1}(\bar{G})$ and $G$ is of class $C^{m}$; then $u(x)$ is a classical solution of (II.1.1).

Finally we have the following result.

LEMMA V.4.3. Let $u(x)$ be a generalized solution of the boundary value problem (II.1.1). Suppose the regularity conditions I(a), (b), (c) are satisfied. Then $\left.u\right|_{\partial G}=\left.D u\right|_{\partial G}=\cdots=\left.D^{m-1} u\right|_{\partial G}=0$ (in the pointwise sense).

Proof. By Lemma V.3.2, $u(x) \in C^{m-1}(\bar{G})$; hence the result follows immediately from Lemma V.4.2.

VI. Spectral theory. In this section, the nonlinear aspect of the eigenvalue problem (II.1.1) is considered. The major nonlinear effect studied is the dependence of the eigenvalue, characterized in Theorem IV.1, on the normalization constant $R$. For convenience, we shall assume throughout this section that the Dirichlet form $a(u, v)$ for (II.1.1) can be associated with a positive definite operator $\mathfrak{A}$ mapping $\mathscr{W}_{2}^{(m)}(G) \rightarrow \mathscr{W}_{2}^{(m)}(G)$. The polyharmonic operator $(-1)^{m} \Delta^{m}$ is a case in point. As $\lambda=\langle\mathfrak{A} u, u\rangle_{m, 2} /\langle B u, u\rangle_{m, 2}$, the positive definiteness of $\mathfrak{A}$ insures that all possible eigenvalues of (II.1.1) are positive. The material in this section is a direct generalization of Levinson [23].

VI.1. Examples. The following examples indicate directions for more general results.

EXAMPle VI.1.1 (The LINEAR CASE). Here $f(u, x)=g(x) u$, where $g(x)$ is a continuous and strictly positive function defined on $G$. By the existence theorem of $\S \mathrm{IV}$ and its corollary, a first eigenvalue $\lambda_{1}$ exists on $\partial M_{R}$ for fixed $R, 0<R<\infty$. Further

$$
\lambda_{1}=\frac{\left\langle\mathfrak{A} u_{1}, u_{1}\right\rangle_{m, 2}}{\int_{G} g(x) u_{1}^{2}}, \text { where } 2 R=\int_{G} g(x) u_{1}^{2} .
$$

As $R$ ranges over $(0, \infty), \lambda_{1}=\lambda_{1}(R)$ remains constant.

EXAMPle VI.1.2 (The CASE OF POWER NONLINEARITIES). Here $f(u, x)=g(x) u^{2 k+1}$, where $g(x)$ is a continuous strictly positive function in $G$, and $k$ is an integer with 


$$
\begin{array}{ll}
2 k+1<\frac{N+2 m}{N-2 m}, & N>2 m, \\
2 k+1<\infty, & N \leqq 2 m .
\end{array}
$$

By the existence theorem of $\S \mathrm{IV}$ and its corollary, a first eigenvalue $\lambda_{1}$ exists for (II.1.1) with $\lambda_{1}=\left\langle\mathfrak{Y} u_{1}, u_{1}\right\rangle_{m, 2} / \int_{G} g(x) u^{2 k+2}$ when $(2 k+2) R=\int_{G} g(x) u^{2 k+2}$. Setting $u_{1}=c v_{1}, c>0$, in (II.1.1) we obtain $A v_{1}-\lambda c^{2 k} f\left(v_{1}, x\right)=0$. Thus for each constant $c>0, v_{1}$ is an eigenfunction for (II.1.1) with eigenvalue $\lambda c^{2 k}$. As $c$ varies over $(0, \infty), \lambda_{1} c^{2 k}$ varies over $(0, \infty)$.

EXAMPLE VI.1.3 (The CASE OF EXPONENTIAL-GROWTH NONLINEARITIES). Following Bratu [4] we consider the two point boundary value problem:

$$
\begin{aligned}
u^{\prime \prime}+\lambda e^{u} & =0, \\
u(a) & =u(b)=0
\end{aligned}
$$

when $a$ and $b$ are fixed finite numbers. Bratu proved the following results: if $\lambda \leqq 0$, (1) has no real solution. There is a fixed positive number $\lambda_{1}$, such that for each $\lambda$, $0<\lambda<\lambda_{1}$, (1) has precisely two positive solutions. If $\lambda=\lambda_{1}$, (1) has precisely one positive solution. For each $\lambda>\lambda_{1}$, (1) again has no real solution. Thus the "spectrum" of the equation (1) consists of the open interval $\left(0, \lambda_{1}\right)$. Further as $\lambda \rightarrow 0,\left\|u_{i}\right\|_{c[a, b]} \rightarrow 0$ when $i=1,2$ and $u_{1}, u_{2}$ are the solutions referred to by Bratu. We shall fit this example into our theory in $\S \mathrm{VII} .2$ by considering $v(x)$ $=u_{1}(x)-u_{2}(x)$ and the equation $v^{\prime \prime}+\lambda g(x)\left[e^{v}-1\right]=0, v(a)=v(b)=0$, where $g(x)$ is a strictly positive continuous function in $[a, b]$ (cf. Gel'fand [39]).

EXAMPLE VI.1.4 (THE CASE OF OSCILlATORY NONLINEARITIES). We consider the two point boundary value problem:

$$
\begin{aligned}
u^{\prime \prime}+\lambda u \sin \log u^{2} & =0, \quad \sin \log 0=0, \\
u(a) & =u(b)=0 .
\end{aligned}
$$

By the results of Golomb [15], a real eigenvalue $\lambda_{0}$ and associated real eigenfunction $u_{0}$ are known to exist. Hence for every integer $N$, the functions $\left\{e^{N \pi} u_{0}(x)\right\}$ are again eigenfunctions for (1) with associated eigenvalue $\lambda_{N}=\lambda_{0}$, and the functions $\left\{e^{(N+1 / 2) \pi} u_{0}(x)\right\}$ are also eigenfunctions for (1) with associated eigenvalue $\lambda_{N}=-\lambda_{0}$. We have omitted such nonlinearities from our discussion.

VI.2. Instances with eigenvalues bounded above 0 . We begin our study by demonstrating that in at least two cases all possible eigenvalues of (II.1.1) are bounded above 0 , i.e. greater than a certain positive constant $k_{0}$.

First suppose $f(t, x)$ is weakly nonlinear, i.e. satisfies a global Lipschitz condition in $t \in R^{\prime}$. Then

Next, we have:

$$
\lambda=\frac{\langle\mathfrak{I} u, u\rangle_{m, 2}}{\int_{G} u f(u, x)} \geqq \frac{k\|u\|_{m, 2}^{2}}{c\|u\|_{0,2}^{2}} \geqq \frac{k}{c}>0 .
$$


Lemma VI.2.1. Let $R$ be a fixed positive constant. Then on $\partial M_{R}$, all possible eigenvalues of (II.1.1) are bounded above 0 . If $\mathfrak{A}$ is not necessarily positive definite, then all eigenvalues are greater than a negative constant, i.e. bounded above $-\infty$.

Proof. Let us first assume $\mathfrak{A}$ is positive definite; then $\langle\mathfrak{A} u, u\rangle_{m, 2} \geqq k_{1}\|u\|_{m, 2}^{2}$ for $k_{1}>0$. On $\partial M_{R}$, for fixed $R$, by Lemma III.2.1, $\|u\|_{m, 2}$ is bounded above 0; thus, on $\left.\partial M_{R},\langle\mathfrak{A} u, u\rangle_{m, 2} \geqq \alpha\right\rangle 0$. On the other hand, by Lemma III.1.1, $\int_{G} u f(u, x) \leqq K \int_{G} F(u, x) \leqq K R$ if $u \in \partial M_{R}$. Hence

$$
\lambda=\frac{\langle\mathfrak{U} u, u\rangle_{m} 2}{\int_{G} u f(u, x)} \geqq \frac{\alpha}{K R}>0 .
$$

If $\mathfrak{A}$ is not necessarily positive definite, by Lemma IV.1.1, $\langle\mathfrak{A} u, u\rangle_{m, 2} \geqq-g(R)$ on $\partial M_{R}$. Thus $\lambda \geqq-g(R) / K R>-\infty$.

VI.3. Auxiliary estimates. Before proceeding further with this discussion, it will be necessary to prove the following two estimates.

LEMMA VI.3.1. Let $R$ be a fixed positive number. Then the following estimate holds:

$$
l(R)=\inf _{\partial M_{R}}\langle\mathfrak{U} u, u\rangle_{m, 2} \leqq \bar{k}(G) g\left(k_{1}(G) R\right)
$$

where $\bar{k}(G)$ and $k_{1}(G)$ are positive constants depending only on the domain $G$ and $g(R)=F^{-1}\left(R, x_{1}\right)(R>0)$.

Proof. To estimate $\langle\mathfrak{A} u, u\rangle_{m, 2}$ we note that as $\mathfrak{A}$ is a bounded operator, $\langle\mathfrak{A} u, u\rangle_{m, 2} \leqq k\|u\|_{m, 2}^{2}$. Hence

$$
l(R) \leqq k\|u\|_{m, 2}^{2}
$$

for any $u \in \partial M_{R}$. We now proceed to construct a function $U_{\theta_{1}}(x) \in \partial M_{R}$ and to compute its $W_{2}^{m}(G)$ norm.

Let $S$ be the radius of the largest open sphere $\mathscr{S}$ completely contained in the interior of $G$. Define

$$
U_{\theta}(x)= \begin{cases}\theta\left(1-\frac{r^{2}}{S^{2}}\right), & r<S, \\ 0, & r \geqq S,\end{cases}
$$

where $r$ is measured in polar coordinates from the center of the sphere $\mathscr{S}$, and $\theta$ is a positive number to be determined. Clearly $\left\|U_{\theta}\right\|_{m, 2}^{2} \leqq K_{G} \theta^{2}$, when $K_{G}$ is a positive constant depending only on the geometry of the bounded domain $G$.

We now show that for some number $\theta_{1}, u_{\theta_{1}} \in \partial M_{R}$. Now $\int_{G} F\left(u_{\theta}, x\right)>$ $\int_{r<s / 2} F\left(u_{\theta}, x\right) \geqq k \int_{r<s / 2} F\left(u_{\theta}, x_{1}\right)>k F\left(3 \theta / 4, x_{1}\right) V$ where $V$ is the volume of the sphere of radius $S / 2$ in $R^{N}$. As $F\left(t, x_{1}\right)$ is a monotone strictly increasing function 
of $t$ from 0 to $\infty$, we can find a number $\theta_{0}$ so that $R=k F\left(3 \theta_{0} / 4, x_{1}\right) V^{(2)}$. Thus $\int_{G} F\left(u_{\theta_{0}}, x\right)>R$ and by the homogeneity lemma there is a number $\theta_{1}<\theta_{0}$ such that $\int_{G} F\left(u_{\theta_{1}}, x\right)=R$, i.e. $u_{\theta_{1}} \in \partial M_{R}$. Also $\left\|u_{\theta_{1}}\right\|_{m, 2}^{2} \leqq \theta_{1}^{2} K(G) \leqq \theta_{0}^{2} K(G)$. Now $\theta_{0}=2 g\left(k_{G} R\right)$ by (2) as $F\left(t, x_{1}\right)$ is an invertible function for positive $t$. From (1), $l(R) \leqq k_{G} g\left(k_{G} R\right)$.

LemMa VI.3.2. Let $R$ be a fixed positive number, and $u_{R}(x)$ be a classical eigenfunction of (II.1.1) as characterized in Theorem IV.1; then as $R \rightarrow 0$, $\left\|u_{R}\right\|_{C(\bar{G})} \rightarrow 0$.

Proof. This result will be obtained by a bootstrap procedure based on Sobolev's Imbedding Theorem and the inequality associated with Theorem V.1.1. First, as $\mathfrak{A}$ is positive definite,

$$
0<k\left\|u_{R}\right\|_{m, 2}^{2} \leqq l(R)=\left\langle\mathfrak{U} u_{R}, u_{R}\right\rangle_{m, 2} .
$$

Thus by Lemma VI.3.1 as $R \rightarrow 0, l(R) \rightarrow 0$ and $\left\|u_{R}\right\|_{m, 2} \rightarrow 0$. Thus by Sobolev's Imbedding Theorem $\|u\|_{0, p} \rightarrow 0$ for

$$
\begin{array}{ll}
1 \leqq p<\frac{N+2 m}{N-2 m}, & N>2 m, \\
1 \leqq p<\infty, & N \leqq 2 m .
\end{array}
$$

Case I $(N \leqq 2 m)$. By Theorem V.1.1,

$$
\left\|u_{R}\right\|_{2 m, p} \leqq k\left(\left\|f\left(u_{R}, x\right)\right\|_{0, p}+\left\|u_{R}\right\|_{0, p}\right)
$$

for every $p, 1<p<\infty$. We now choose $p$ so that $1 / p-2 m / N<0$, and by Sobolev's Imbedding Theorem (part ii):

$$
\left\|u_{R}\right\|_{C(\bar{G})} \leqq k_{1}\left\|u_{R}\right\|_{2 m, p} .
$$

Thus using the polynomial growth condition on $f(t, x)$ together with (1) and (2), we obtain the fact that as $R \rightarrow 0,\left\|u_{R}\right\|_{C(\bar{G})} \rightarrow 0$.

Case II $(N>2 m)$. Again from Theorem V.1.1

$$
\left\|u_{R}\right\|_{2 m, p} \leqq k\left(\left\|f\left(u_{R}\right)\right\|_{0, p}+\left\|u_{R}\right\|_{0, p}\right) .
$$

By Lemma V.1.2 $f\left(u_{R}(x), x\right) \in L_{2 N \cdot(1+\varepsilon) /(N+2 m)}$, thus by the polynomial growth condition on $f(t, x),\left\|f\left(u_{R}, x\right)\right\|_{L_{p}} \leqq K g\left(\|u\|_{2 N /(N-2 m)}\right)$ when $p=2 N \cdot(1+\varepsilon) /(N+2 m)$ and $g(r)$ is a monotone increasing function of the real number $r$. Thus by Sobolev's Imbedding Theorem $\|u\|_{2 m, p} \rightarrow 0$ as $R \rightarrow 0$. By repeating this procedure as in Lemma V.3.3 we eventually obtain $\left\|u_{R}\right\|_{2 m, p_{N}} \rightarrow 0$, where $1 / p_{N^{\prime}}-2 m / N<0$. Hence, by using Sobolev's Imbedding Theorem part (ii), we obtain $\left\|u_{R}\right\|_{C(\bar{G})}$ $\leqq k\left\|u_{R}\right\|_{2 m, p_{N}}$. Hence as $R \rightarrow 0,\left\|u_{R}\right\|_{C(\bar{G})} \rightarrow 0$.

VI.4. Study of the set $\{\lambda(R) \mid 0<R<\infty\}$. For a large class of nonlinear operators $B$, the eigenvalue $\lambda$ of (II.1.1) as characterized in Theorem IV.1.1, 
varies over the interval $(0, \infty)$ as the normalization constant $R$ varies over $(0, \infty)$. In order to demonstrate this result, the following terminology will be convenient.

DefinITION VI.4.1. Let $f(t, x)$ be a continuous real-valued function defined on $R^{\prime} \times G$; then

$f(t, x)$ is supralinear at $t=b(b=0$ or $\infty)$ if

$$
\lim _{t \rightarrow b} \frac{f(t, x)}{t}=\infty \text { for all } x \in G .
$$

$f(t, x)$ is sublinear at $t=b(b=0$ or $\infty)$ if

$$
\lim _{t \rightarrow b} \frac{f(t, x)}{t}=0 \text { for all } x \in G
$$

LEMmA VI.4.1. Suppose $f(t, x)$ is sublinear at $t=\infty$ and a generalized eigenfunction, as characterized in Theorem IV.1 exists, for the boundary value problem (II.1.1). Then as $R \rightarrow \infty, \lambda(R) \rightarrow \infty$.

Proof. As $f(t, x)$ is sublinear at $t=\infty$,

$$
\lim _{t \rightarrow \infty} \frac{F(t, x)}{t^{2}}=0
$$

We show that as $R \rightarrow \infty,\left\|u_{R}\right\|_{L_{2}} \rightarrow \infty$. Select a number $A$ such that $t<A$ implies $F(t, x)<t^{2}$. Therefore

$$
\begin{aligned}
R & =\int_{G} F(u, x)=\int_{|u|>A} F(u, x)+\int_{|u| \leqq A} F(u, x) \\
& \leqq \int_{|u|>A} u^{2}+F(A) \operatorname{meas}(G) \\
& \leqq\|u\|_{L_{2}(G)}^{2}+K(A) .
\end{aligned}
$$

Thus as $R \rightarrow \infty,\|u\|_{L_{2}(G)} \rightarrow \infty$.

As $f(t, x)$ is sublinear at $t=\infty$, there is a number $B$ such that $t>B$ and given $\delta_{1}>0, f(t, x)<\delta_{1} t$. Hence

$$
\begin{aligned}
\int_{G} u f(u, x) & =\int_{|u|>B} u f(u, x)+\int_{|u| \leqq B} u f(u, x) \\
& \leqq \delta \int_{|u|>B} u^{2}+\int_{|u| \leqq B} u f(u, x) \leqq \delta_{1}\|u\|_{L_{2}(G)}^{2}+B \bar{f}(B) \text { meas } G .
\end{aligned}
$$

Since $\mathfrak{A}$ is assumed positive definite there is a positive constant $k$ such that $\langle\mathfrak{A} u, u\rangle_{m, 2} \geqq k\|u\|_{m, 2}^{2}>k\|u\|_{0,2}^{2}$. Thus

$$
\lambda(R)=\frac{\left\langle\mathfrak{A} u_{R}, u_{R}\right\rangle_{m, 2}}{\int_{G} u_{R} f\left(u_{R}, x\right)} \geqq \frac{k\left\|u_{R}\right\|_{0,2}^{2}}{\delta_{1}\left\|u_{R}\right\|_{0,2}^{2}+B \tilde{f}(B) \text { meas } G} .
$$


As $R \rightarrow \infty,\|u\|_{0,2}^{2} \rightarrow \infty$, and as $\delta_{1}$ can be made arbitrarily small, $\lambda(R) \rightarrow \infty$.

LEMMA VI.4.2. Suppose $f(t, x)$ is sublinear at $t=0$, and a classical eigenfunction, as characterized in Theorem IV.1, exists for the boundary value problem (II.1.1); then as $R \rightarrow 0, \lambda(R) \rightarrow \infty$.

Proof. By Lemma VI.3.2 as $R \rightarrow 0, \max _{G}\left|u_{R}(x)\right| \rightarrow 0$. Hence using the fact that $f(t, x)$ is sublinear at $t=0$ given $\delta_{1}>0$, for sufficiently small $R, f\left(u_{R}, x\right)<\delta_{1} u_{R}$. Therefore,

$$
\lambda(R)=\frac{\left\langle\mathfrak{A} u_{R}, u_{R}\right\rangle_{m, 2}}{\int_{G} u_{R} f\left(u_{R}, x\right)} \geqq \frac{k\left\|u_{R}\right\|_{0,2}^{2}}{\delta_{1}\left\|u_{R}\right\|_{0,2}^{2}} \geqq \frac{k}{\delta_{1}} .
$$

As $\delta_{1}$ can be made arbitrarily small, $\lambda(R) \rightarrow \infty$ as $R \rightarrow 0$.

LEMMA VI.4.3. Suppose $f(t, x)$ is supralinear at $t=\infty$, and a generalized eigenfunction, as characterized in Theorem IV.1 exists for (II.1.1). Then as $R \rightarrow \infty, \lambda(R) \rightarrow 0$.

Proof. As $f(t, x)$ is supralinear at $t=\infty$,

$$
\lim _{t \rightarrow \infty} \frac{F(t, x)}{t^{2}}=\infty
$$

Thus given $\delta_{1}>0$ for sufficiently large $\theta_{0}, F\left(3 \theta_{0} / 4, x_{1}\right)>\theta_{0}^{2} / \delta_{1}$. By LemmaVI.3.1,

$$
R=\int_{G} F\left(u_{\theta_{0}}, x\right) \geqq \int_{r \leqq S / 2} F\left(u_{\theta_{0}}, x\right) \geqq F\left(\frac{3 \theta_{0}}{4}, x_{1}\right) k(G) .
$$

Thus $R>k(G) \theta_{0}^{2} / \delta$, where $R$ is sufficiently large. Also $\int_{G} u f(u, x) \geqq k \int_{G} F(u, x)$

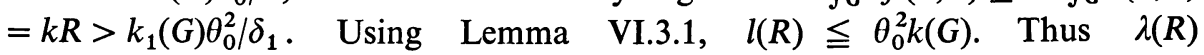
$=l(R) / \int_{G} u f(u, x) \leqq k_{2}(G) \delta_{1}$.

As $\delta_{1}$ can be made arbitrarily small as $R \rightarrow \infty, \lambda(R) \rightarrow 0$.

LEMMA VI.4.4. Suppose $f(t, x)$ is supralinear at $t=0$, and a classical eigenfunction, as characterized in Theorem IV.1 exists, for (II.1.1). Then as $R \rightarrow 0$, $\lambda(R) \rightarrow 0$.

Proof. As $f(t, x)$ is supralinear at $t=0$

$$
\lim _{t \rightarrow 0} \frac{F(t, x)}{t^{2}}=\infty \text {. }
$$

Thus given $\delta_{1}>0$, we can choose a $\theta_{0}$ so small that $F\left(\theta_{0} / 2, x_{1}\right) \geqq k(G) \theta_{0}^{2} / \delta_{1}$. Hence proceeding as in Lemma VI.4.3, we can conclude as $R \rightarrow 0, \lambda(R) \rightarrow 0$.

One consequence of the above lemmas is the following theorem.

THEOREM VI.4.5. Suppose $f(t, x)$ is supralinear at one end of the interval $(0, \infty)$ and sublinear an the other. Suppose a classical eigenfunction, as charac- 
terized in Theorem IV.1, exists for (II.1.1). Then the set of positive numbers $\{\lambda(R) \mid 0<R<\infty\}$ contains subsequences tending both to 0 and $+\infty$.

VI.5. Further study of the set $\{\lambda(R) \mid 0<R<\infty\}$. We bring this section to a close by considering circumstances under which the set $\{\lambda(R) \mid 0<R<\infty\}$ fills an interval of the positive axis (cf. VI.1). It is sufficient to prove $\lambda=\lambda(R)$ is a continuous function of $R$.

LEMMA VI.5.1. $l(R)=\inf _{\partial M_{R}}\langle\mathfrak{A} u, u\rangle_{m, 2}$ is a continuous monotone increasing function of $R$ for $0<R<\infty$.

Proof. First we show that $l(R)$ is monotone increasing. Let $R>R_{1}$ and $u \in \partial M_{R}$. Then by the homogeneity property of $\partial M_{R}$, there is a number $b<1$, such that $b u \in \partial M_{R_{1}}$. Thus $\langle\mathfrak{U}(b u), b u\rangle_{m, 2} \geqq l\left(R_{1}\right)$. If $u$ is actually a solution of the variational problem on $\partial M_{R}$ we have $\langle\mathfrak{U}(b u), b u\rangle_{m, 2}=b^{2} l(R) \geqq l\left(R_{1}\right)$. As $b<1$, $l(R)>l\left(R_{1}\right)$.

Next we show $l(R) \geqq l(R+0)$. Let $R>R_{1}$ and $u_{1} \in \partial M_{R_{1}}$; thus there is a number $b>1$ such that $b^{2}\left\langle\mathfrak{U} u_{1}, u_{1}\right\rangle_{m, 2} \geqq l(R)$. By Lemma III.2.2, $R \rightarrow R_{1}$ implies $b \rightarrow 1$. In particular if $u_{1}$ is a solution of the variational problem on $\partial M_{R_{1}}$, $b^{2} l\left(R_{1}\right) \geqq l(R)$. Thus $l(R) \geqq l(R+0)$.

Finally $l(R-0) \geqq l(R)$. Let $R_{n}$ be an increasing sequence of positive numbers tending to $R$, with associated minimum function $u_{n}$. Thus by the homogeneity property of $\partial M_{R}$, there is a sequence of positive numbers $\left\{b_{n}\right\}, b_{n} \geqq 1$, such that $b_{n} u_{n} \in \partial M_{R}$. Thus $b_{n}^{2} l\left(R_{n}\right) \geqq l(R)$. In order to show $l(R-0) \geqq l(R)$ it is sufficient to show $b_{n} \rightarrow 1$. To this end, we consider

$$
0=\lim _{n \rightarrow \infty} \int_{G}\left[F\left(b_{n} u_{n}, x\right)-F\left(u_{n}, x\right)\right] .
$$

By Fubini's theorem and Lemma III.1.2

$$
0=\lim _{n \rightarrow \infty} \int_{0}^{1} d t \int_{G}\left(b_{n}-1\right) u_{n} f\left[u_{n}\left[1+t\left(1-b_{n}\right)\right], x\right] .
$$

As $t f(t, x)>0, t \neq 0$, this latter equation is possible only if $b_{n} \rightarrow 1$.

LEMMA VI.5.2. Suppose $\alpha(R)=\left\langle u_{R}, B u_{R}\right\rangle_{m, 2}$ is a well-defined function of the positive number $R$, where $u_{R} \in \partial M_{R}$ is a solution of the variational problem $\inf _{\partial M_{R}}\langle\mathfrak{U} u, u\rangle_{m, 2}$. Then $\alpha(R)$ is a continuous function of $R$.

Proof. Let $R_{n} \rightarrow R$ and consider $\alpha\left(R_{n}\right)=\left\langle u_{n}, B u_{n}\right\rangle_{m, 2}$ where $u_{n}$ is an associated minimum function of $R_{n}$. It is sufficient to show $\left\{R_{n}\right\}$ has a subsequence $\left\{R_{n^{\prime}}\right\}$ such that $\alpha\left(R_{n^{\prime}}\right) \rightarrow \alpha(R)$.

First we note, for sufficiently large $n, 0<\int_{G} u_{n} f\left(u_{n}, x\right)<K \int_{G} F\left(u_{n}, x\right)<K R$ $<K(R+1)$. Thus the set of positive numbers $\left\langle u_{n}, B u_{n}\right\rangle_{m, 2}$ is uniformly bounded and has a convergent subsequence. As the form $\langle u, B v\rangle_{m, 2}$ is a weakly continuous 
function of the elements $u, v$ it is sufficient to show $\left\{u_{n}\right\}$ has a subsequence $u_{n^{\prime}}$ such that $u_{n^{\prime}} \rightarrow u_{R}$ weakly in $\mathscr{W}_{2}^{(m)}(G)$.

Next by the homogeneity property of $\partial M_{R}$, there is a sequence of real numbers $\left\{b_{n}\right\}$ such that $b_{n} u_{n} \in \partial M_{R}$. We show $\left\{b_{n} u_{n}\right\}$ is a minimizing sequence for the variational problem $\inf _{\partial M_{R}}\langle\mathfrak{A} u, u\rangle_{m, 2}$,

$$
\lim _{n \rightarrow \infty}\left\langle\mathfrak{U}\left(b_{n} u_{n}\right), b_{n} u_{n}\right\rangle_{m, 2}=\lim _{n \rightarrow \infty} b_{n}^{2} l\left(R_{n}\right) .
$$

By the argument of Lemma VI.5.1, $b_{n} \rightarrow 1$ and $l\left(R_{n}\right) \rightarrow l(R)$; hence $\lim _{n \rightarrow \infty}\left\langle\mathfrak{U}\left(b_{n} u_{n}\right), b_{n} u_{n}\right\rangle_{m, 2}=l(R)$ and the sequence $\left\{b_{n} u_{n}\right\}$ has the required property.

Now by the procedure of §IV the minimizing sequence can be refined so that there is a subsequence $\left\{b_{n^{\prime}} u_{n^{\prime}}\right\}$ tending weakly to $u_{R}$. As $b_{n^{\prime}} \rightarrow 1, u_{n^{\prime}} \rightarrow u_{R}$ weakly in $\mathscr{W}_{2}^{(m)}(G)$, as required.

REMARK. $\alpha(R)$ is a well-defined function of $R$ if either (a) the variational problem $\inf _{\partial M_{R}}\langle\mathfrak{A} u, u\rangle_{m, 2}$ has a unique solution, apart from sign, as one expects for ordinary differential equations, or (b)

$$
\int_{G} u f(u, x)=K \int_{G} F(u, x) \text { for all } u \in \mathscr{W}_{2}^{(m)}(G)
$$

for some constant $K$.

We summarize the results of this subsection as follows.

THEOREM VI.5.3. Suppose the variational problem $\inf _{\partial M_{R}}\langle\mathfrak{A} u, u\rangle_{m, 2}$ has a unique solution for each $0<R<\infty$ (apart from sign); then $\lambda(R)$ is a continuous function of $R$.

Proof. $\lambda(R)=\langle\mathfrak{A} u, u\rangle_{m, 2} /\langle B u, u\rangle_{m, 2}=l(R) / \alpha(R)$ and, for $0<R<\infty$ is the quotient of two continuous functions by Lemmas VI.5.1-2. Thus $\lambda(R)$ is a continuous function, as $\alpha(R) \neq 0$ for $0<R<\infty$.

VII. Second order equations and the case $2 m>N$. In this last section, the existence Theorem IV.1 will be sharpened in two ways. First, we investigate the positivity properties of the eigenfunctions obtained. Second, we mention some cases in which Theorem IV.1 holds for nonlinearities determined by a function $f(t, x)$ which does not satisfy a polynomial growth condition.

Throughout $\S$ VII.1 we consider the boundary value problem (II.1.1) in the special case of second order operators $A$ :

$$
A u-\lambda f(u, x)=0
$$

$$
\left.u\right|_{\partial G}=0 \text {, }
$$

when $A u=\sum_{|\alpha|,|\beta| \leqq 1} D^{\alpha}\left(a_{\alpha \beta}(x) D^{\beta} u\right)$.

As $A$ is formally self-adjoint, $a_{0 \alpha}=a_{\beta 0} \equiv 0$ for $\alpha, \beta \neq 0$. Also, in this case the 
associated operator $\mathfrak{A}: \mathscr{W}_{2}^{(1)}(G) \rightarrow \mathscr{W}_{2}^{(1)}(G)$ is positive definite, as $A$ is uniformly elliptic in $G$.

VII.1. Positivity properties of eigenfunctions. The following result gives a partial extension of the Courant-Nodal Line Theorem to a nonlinear context (cf. Courant-Hilbert [9, Vol. I, p. 451]).

THEOREM VII.1.1 Let the hypotheses of Theorem IV.1 be satisfied. Then the boundary value problem (VII.1) has a generalized eigenfunction $u(x) \geqq 0$ in $G$. If, in addition, $u(x)$ is a classical eigenfunction for (VII.1), then $u(x)>0$ in $G$.

Proof. First we note that as $F(t, x)$ is an even function of $t, \int_{G} F(u, x)$ $=\int_{G} F(|u|, x)$. Also $|u(x)|=\operatorname{sgn} u \cdot u$. Hence if $u \in \mathscr{W}_{2}^{(1)}(G), D_{j}|u(x)|$ $=\operatorname{sgn} u \cdot D_{j} u(x)$ a.e. in $G$, and $|u| \in \mathscr{W}_{2}^{(1)}(G)$. Hence if $u \in \partial M_{R},|u| \in \partial M_{R}$.

As $A$ is formally self-adjoint, $a_{\alpha 0}=a_{0 \beta} \equiv 0$ if $\alpha, \beta \neq 0$; thus

$$
\begin{aligned}
\langle\mathfrak{A}|u|,|u|\rangle_{1,2} & =\sum_{|\alpha|,|\beta| \leqq 1} \int_{G} a_{\alpha \beta}(x) D^{\alpha}|u| D^{\beta}|u| \\
& =\sum_{|\alpha|,|\beta| \leqq 1} \int_{G} a_{\alpha \beta}(x) D^{\alpha} u D^{\beta} u=\langle\mathfrak{A} u, u\rangle_{1,2} .
\end{aligned}
$$

Thus to prove the first part we need only follow the procedure of §IV with the additional requirement of choosing the minimizing sequence $\left\{u_{n}\right\}$ from the nonnegative functions $u(x) \geqq 0$ in $G$. As $\langle\mathfrak{A}|u|,|u|\rangle_{1,2}=\langle\mathfrak{A} u, u\rangle_{1,2}$ the selection of a minimizing sequence of nonnegative functions from $\partial M_{R}$ does not affect the variational problem $\inf _{\partial M_{R}}\langle\mathfrak{A} u, u\rangle_{1,2}$. By the refinement procedure of Lemma IV.1.3 the minimizing sequence $u_{n}(x) \rightarrow u(x)$ a.e. in $G$. Thus, after possible correction on a set of measure 0 , we may assume $u(x) \geqq 0$ in $G$.

We now consider the second part of the theorem. If $u(x) \geqq 0$ in $G, f(u, x) \geqq 0$ in $G$ and as $\mathfrak{A}$ is positive definite $\lambda \geqq 0$. Thus if $u$ is a classical solution of (VII.1), $-A u=-\lambda f(u, x) \leqq 0$. Thus by Hopf's Maximum Theorem, see [9], $u(x)>0$ in $G$, as $\min _{\partial G} u=0$.

REMARK. If $u(x)$ is a generalized eigenfunction of VII.1, $u(x)$ cannot equal 0 on a set of measure greater than 0 in $G$ (cf. Gilbarg [14]).

COROllary to TheOREM VII.1.1. Theorem VII.1.1 holds without the following two assumptions on $f(t, x)$ :

$$
\begin{aligned}
f(-t, x) & =-f(t, x), \\
t f(t, x) & >0 \quad(t \neq 0) .
\end{aligned}
$$

Proof. Define

$$
f^{*}(t, x)= \begin{cases}f(t, x), & t \geqq 0 \\ -f(-t, x), & t<0\end{cases}
$$


Thus $f^{*}(t, x)$ satisfies all the requirements of the existence Theorem IV.1. Thus by Theorem VII.1.1 there is a function $u(x) \geqq 0$ such that

$$
\begin{aligned}
A u-\lambda f^{*}(u, x) & =0, \\
\left.u\right|_{\partial G} & =0
\end{aligned}
$$

(in the classical or generalized sense). By the definition of $f^{*}$ we have $A u-\lambda f(u, x)=0,\left.u\right|_{\partial G}=0$, as required.

VII.2. Sharpening of the polynomial growth condition. Now we turn to the second object of this section, the sharpening of the existence Theorem IV.1 for nonlinearities determined by a function $f(t, x)$ not satisfying a polynomial growth condition.

The best result in this direction is obtained for second order ordinary differential equations and requires no growth restriction on $f(t, x)$.

THeOREM VII.2.1 (GolomB). Let $f(t, x)$ be a continuous real-valued function defined on $R^{\prime} \times[a, b]$, where $a, b$ are finite numbers, such that $f(0, x) \equiv 0$ and, for $t \neq 0, f(t, x) \neq 0$. Then the ordinary differential equation

$$
\begin{aligned}
\left(p(x) u^{\prime}\right)^{\prime}+\lambda f(u, x) & =0, \\
u(a) & =u(b)=0,
\end{aligned}
$$

has a real classical eigenfunction $u(x)$ and real eigenvalue when $p(x)$ is a continuous strictly positive function on $[a, b]$.

The proof of this result is contained in the paper of Golomb [15]. The following theorem extends Golomb's result to cases in which $2 m>N$ and, in particular, to all even order ordinary differential equations.

THEOREM VII.2.2. In case $2 m>N$, the existence Theorem IV.1 and the regularity Theorem V.1 hold without any growth restriction on the function $f(t, x)$.

Proof. This result is immediate if we repeat $\S \S I I$, III, V, using the fact that when $2 m>N$, by Sobolev's Imbedding Theorem $W_{2}^{m}(G) \subset C^{0 h}(G)$ (with a compact imbedding). This fact replaces the polynomial growth restriction on $f(t, x)$. For example, in Lemma II.3.3 we must prove

$$
\sup _{\|v\| \leqq 1} \int_{G}\left(f\left(u_{n}, u\right)-f(u, x)\right) v \rightarrow 0
$$

where $u_{n} \rightarrow u$ weakly in $W_{2}^{m}(G)$. Hence $u_{n} \rightarrow u$ strongly in $C(G)$; thus $u_{n}$ is uniformly bounded by $M$, say, $\left|f\left(u_{n}, x\right)\right| \rightarrow|f(u, x)|$ and $\left|f\left(u_{n}, x\right)\right| \leqq|f(M, x)|$; hence as $n \rightarrow \infty$, (1) holds. Again in Lemma III.2.1, to prove $M_{R}$ is uniformly bounded away from 0 , we suppose the contrary; then there is a weakly convergent sequence $u_{n} \in M_{R}$ with $\left\|u_{n}\right\|_{m, 2} \rightarrow 0$. But then 


$$
\begin{aligned}
0<R & =\int_{G} F\left(u_{n}, x\right) \leqq K \int_{G} u_{n} f\left(u_{n}, u\right) \leqq K \max _{G}\left|u_{n}(x)\right| \int_{G} f\left(u_{n}, x\right) \\
& \leqq K K^{\prime}\left\|u_{n}\right\|_{m, 2} \int_{G} f\left(u_{n}, x\right) \quad \text { (by Sobolev Imbedding Theorem) } \\
& \left.\leqq K K^{\prime}\left\|u_{n}\right\|_{m, 2} \int_{G} f\left(M^{\prime}, x\right) \quad \text { (where } M^{\prime}=\max _{G}\left|u_{n}(x)\right|\right) .
\end{aligned}
$$

As this latter expression tends to zero as $n \rightarrow \infty$, we obtain the required contradiction. The variational lemma carries over immediately when we note the elementary fact that a continuous function defined on a closed (bounded set in $R^{N}$ ) is uniformly continuous. The regularity theorem V.1 carries over by using theorem V.2.2 immediately instead of beginning with $\S \mathrm{V} .1$.

For $A=-\Delta$, the Laplace operator in $R^{2}$, Levinson [23], proves the following:

THEOREM VII.2.3. Let $G$ be a bounded domain in $R^{2}$ with boundary $G$ consisting of a finite number of continuous curves and arcs. Suppose $f(t)$ is a continuous real-valued function defined on $[0, \infty)$ and vanishing only at $t=0$. Suppose, in addition, that $f(t)$ satisfies a local Lipschitz condition, is nondecreasing and satisfies the following growth condition:

$$
f(t) \log f(t) \leqq K^{\prime} \int_{0}^{t} f(s) d s
$$

for $t>0$ and some constant $K^{\prime} \geqq 1$. Then the boundary value problem

$$
\begin{aligned}
\Delta u+\lambda f(u) & =0, \\
\left.u\right|_{\partial G} & =0
\end{aligned}
$$

has a real classical eigenfunction $u(x)$, strictly positive in the interior of $G$.

This result represents a first step in establishing exponential type growth conditions on $f(t, x)$ in the case $2 m=N$ (e.g., $f(t)=e^{\sqrt{ } t}-1$ ). The details of this aspect of the problem will be carried out in subsequent work.

\section{BIBLIOGRAPHY}

1. S. Agmon, The $L_{p}$ approach to the Dirichlet problem, Ann. Scuola Norm. Sup. Pisa 13 (1959), 405-448.

2. S. Agmon, A. Douglis and L. Nirenberg, Estimates near the boundary for solutions of elliptic equations, Comm. Pure Appl. Math. 12 (1959), 623-727.

3. S. Banach, Uber homogene polynome in $L^{2}$, Studia Math. 7 (1938), 36-44.

4. G. Bratu, Sur les équations integrales non-linéaires, Bull. Soc. Math. France 42 (1914), 113-142.

5. F. E. Browder, On the spectral theory of elliptic differential operators. I, Math. Ann. 142 (1961), 22-130. 81-226.

6. - Functional analysis and partial differential equations. II, Math. Ann. 145 (1962), 
7. - Nonlinear elliptic boundary value problems, Bull. Amer. Math. Soc. 69 (1963), 862-874.

8. - Variational boundary value problems for quasilinear elliptic equations. I, II, III, Proc. Nat. Acad. Sci. U.S.A. 50 (1963), 31-37, 592-598, 794-798.

9. R. Courant and D. Hilbert, Methods of mathematical physics, Vols. 1, 2, New York, 1953, 1962.

10. J. Dubinskii, Some imbedding theorems in Orlicz spaces, Dokl. Akad. Nauk SSSR 152 (1963), 529-532; English transl., Amer. Math. Soc., Providence, R. I., 1963.

11. G. F. D. Duff, Modified boundary value problems for a quasi-linear elliptic equation, Canad. J. Math. 8 (1956), 203-219.

12. N. Dunford and J. T. Schwartz, Linear operators, Vols. 1 and 2, Interscience, New York, 1958, 1963.

13. L. Gårding, Dirichlet's problem for linear elliptic partial differential equations, Math. Scand. 1 (1953), 55-72.

14. D. Gilbarg, Some local properties of elliptic equations, Proc. Sympos. Pure Math. Vol. 4, pp. 127-142, Amer. Math. Soc., Providence, R. I., 1961.

15. M. Golomb, Zur theorie der nichtlinearen Integralgleichungen, Math. Z. 39 (1934), 45-75.

16. A. Granas, Introduction to the toplogy of function spaces, Lecture notes, Univ. of Chicago, Chicago, Ill., 1961.

17. A. Hammerstein, Nichtlineare Integralgleichungen nebst Anwendunger, Acta. Math. 54 (1930), 117-176.

18. G. H. Hardy, J. E. Littlewood and G. Pólya, Inequalities, Cambridge Univ. Press, London, 1934.

19. A. I. Koshelev, A priori estimates in $L_{p}$ and generalized solutions of elliptic eqations and systems, Uspehi Mat. Nauk 13 (1958), 29-88; Amer. Math. Soc. Transl. (2) 20 (1962), 105-171.

20. M. A. Krasnoselskii and Y. Rutitskii, Convex functions, Noordhoff, Groningen, 1961.

21. M. Krein and M. A. Rutman, Linear operarors leaving invariant a cone in a Banach space, Uspehi Mat. Nauk 23 (1948), 3-95; Amer. Math. Soc. Transl. (1) 10 (1962), 199-325.

22. O. A. Ladyzenskaya, Investigation of the Navier-Stokes equation for a stationary flow, Uspehi Mat. Nauk 14 (1959), 75-97; Amer. Math. Soc. Transl., (2) 25 (1963), 173-197.

23. N. Levinson, Positive eigenfunctions for $\Delta u+\lambda f(u)=0$, Arch. Rational Mech. Anal. 11 (1962), 258-272.

24. J. L. Lions, Équations différentialles operationnelles, Springer, Göttingen, 1961.

25. L. Ljusternik, Sur une class d'équations différentielles non-lineaires, Mat.Sb. 44 (1937), 1143-1168.

26. - On a class of non-linear operators in Hilbert space, Izv. Akad. Nauk. SSSR (1939), 257-264.

27. Z. Nehari, Characteristic values associated with a class of non-linear second order differential equations, Acta Math. 105 (1961), 141-175.

28. L. Nirenberg, Estimates and existence of solutions of elliptic equations, Comm. Pure Appl. Math. 9 (1956), 509-530.

29. —_ Remarks on strongly elliptic partial diffrential equations, Comm. Pure Appl. Math. 8 (1955). 649-672.

30. G. Pimbley, A super-linear Sturm-Liouville problem, Trans. Amer. Math. Soc. 103 (1962), 229-248.

31. F. Riesz and B. Sz-Nagy, Functional analysis, Translated by Leo F. Boron, Ungar, New York, 1955. 
32. H. H. Schaeffer, Some non-linear eigenvalue problems, Non-Linear Problems Conference Univ. of Wisconsin, Madison, Wis., 1963.

33. L. Schwartz, Théorie des distributions. II, Hermann, Paris, 1951.

34. M. Vainberg, On the continuity of some operators of special type, Dokl. Akad. Nauk SSSR 73 (1950), 253-255.

35. - On the existence of characteristic functions for non-linear integral equations with non-positive kernels, Dokl. Akad. Nauk SSSR 78 (1951), 1077-1080. (Russian)

36. - Variational methods for the investigation of non-linear operators, GITTL, Moscow, 1956.

37. M. I. Višik, Boundary value problems for quasilinear strongly elliptic systems, Dokl. Akad. Nauk SSSR 138 (1961), 518-521.

38. A. Wintner, The Hölder restrictions on elliptic differential equations, Amer. J. Math. 72 (1950), 731-738.

39. I. M. Gel'fand, Some problems in the theory of quasi-linear equations, Uspehi Mat. Nauk SSSR 14 (1959), 87-158; Amer. Math. Soc. Transl. 29 (1963), 295-381.

YALE UNIVERSITY,

New Haven, Connecticut

UNIVERSITY OF MiNNESOTA,

MinNEAPOLIS, MinNesota 